\title{
Ultralight dark matter in disk galaxies
}

\author{
Nitsan Bar, ${ }^{1, *}$ Kfir Blum, ${ }^{1,2,}$ Joshua Eby, ${ }^{1, *}$ and Ryosuke Sato ${ }^{3, \S}$ \\ ${ }^{1}$ Weizmann Institute of Science, Rehovot 7610001, Israel \\ ${ }^{2}$ Theory department, CERN, CH-1211 Geneve 23, Switzerland \\ ${ }^{3}$ Deutsches Elektronen-Synchrotron (DESY), Notkestraße 85, D-22607 Hamburg, Germany
}

(Received 14 March 2019; published 29 May 2019)

\begin{abstract}
Analytic arguments and numerical simulations show that bosonic ultralight dark matter (ULDM) would form cored density distributions ("solitons") at the center of galaxies. ULDM solitons offer a promising way to exclude or detect ULDM by looking for a distinctive feature in the central region of galactic rotation curves. Baryonic contributions to the gravitational potential pose an obstacle to such analyses, being (i) dynamically important in the inner galaxy and (ii) highly nonspherical in rotation-supported galaxies, resulting in nonspherical solitons. We present an algorithm for finding the ground-state soliton solution in the presence of stationary nonspherical background baryonic mass distribution. We quantify the impact of baryons on the predicted ULDM soliton in the Milky Way and in low-surface-brightness galaxies from the SPARC database.
\end{abstract}

DOI: $10.1103 /$ PhysRevD.99.103020

\section{INTRODUCTION}

An ultralight bosonic field oscillating around a minimum of its potential [1-4] can play the role of dark matter (DM). On cosmologically large scales, ultralight dark matter (ULDM) behaves similarly to cold weakly interacting massive particle (WIMP) dark matter, reproducing its success with respect to the cosmic microwave background and large-scale structure. On smaller scales comparable to the de Broglie wavelength, ULDM behaves differently than WIMPs. In particular, at the center of galactic halos, ULDM develops cored density profiles that lead to markedly different predictions than those found for ordinary WIMPs [1,5-30]. The cored ULDM distributions correspond to quasistationary minimum energy solutions of the equations of motion. We will follow common convention and refer to these solutions as "solitons."

Reference [23] analyzed the rotation curves of wellresolved low-surface brightness (LSB) disk galaxies from the SPARC database [31] and pointed out that these galaxies fail to show the soliton feature predicted by

\footnotetext{
*nitsan.bar@weizmann.ac.il

†kfir.blum@cern.ch

*joshaeby@gmail.com

${ }^{8}$ ryosuke.sato@desy.de
}

Published by the American Physical Society under the terms of the Creative Commons Attribution 4.0 International license. Further distribution of this work must maintain attribution to the author(s) and the published article's title, journal citation, and DOI. numerical simulations $[9,10,32] .{ }^{1}$ This led to the bound $m \gtrsim 10^{-21} \mathrm{eV}$. A similar constraint ${ }^{2}$ was found in Ref. [26] considering the dwarf spheroidal galaxy Eridanus-II. The matter power spectrum inferred from $\mathrm{Ly}-\alpha$ forest analyses yields a comparable bound [34-38]. ${ }^{3}$ These lower bounds on $m$ are interesting because they probe DM using gravity alone, without requiring any direct interactions with SM fields; because they define how light DM could possibly be; and also because ULDM with $m \sim\left(10^{-22}-10^{-21}\right) \mathrm{eV}$ was suggested as an explanation for puzzles facing the WIMP paradigm on small scales $[16,47]$.

In the attempt to constrain (or detect) ULDM with galactic kinematics, an important issue is the modeling of the baryonic contribution to the gravitational potential which can distort the soliton. ${ }^{4}$ Reference [23] analyzed the solution in the presence of a spherically symmetric background potential, in order to estimate the size of the effect. That was found to be significant for the Milky Way (MW) but not significant for the relevant SPARC LSB galaxies.

\footnotetext{
${ }^{1}$ Reference [33] reports independent evidence against soliton cores.

${ }^{2}$ Reference [26] notes that dynamics of a central star cluster in Eridanus-II could potentially probe ULDM up to $m \sim 10^{-19} \mathrm{eV}$.

${ }^{3}$ See also Refs. [39,40]. A bound, $m \gtrsim 10^{-23} \mathrm{eV}$, comes from scalar metric perturbations induced by ULDM [41] that were searched for in pulsar timing signals [42]. Heating of the MW disk suggests $m>0.6 \times 10^{-22} \mathrm{eV}$ [43]. More tentative constraints include $m>1.5 \times 10^{-22} \mathrm{eV}$ [44], based on preliminary analysis of stellar streams in the Milky Way, and $m>8 \times 10^{-21}$ [45], assuming that $21 \mathrm{~cm}$ results by EDGES [46] are confirmed.

${ }^{4}$ See Ref. [48] for a preliminary study of the dynamical impact of stars in ULDM numerical simulations.
} 
However, both in the MW and in rotation-supported SPARC galaxies, the baryonic mass distribution is nonspherical, following disklike morphology. In a nonspherical system, dynamics in the central region of the galaxy can be affected by the mass distribution at larger radii. It is therefore important to extend the soliton + baryon analysis to nonspherical configurations.

In this paper, we present an algorithm to calculate the soliton solution in the presence of a nonspherical background gravitational potential. The algorithm is simple, fast, and accurate and can replace the standard onedimensional shooting method used for solving the spherically symmetric soliton.

Our goals in presenting this tool are twofold. First, it allows us to perform a self-consistent analysis of the velocity profile in disk galaxies. Once the baryonic mass distribution is specified (presumably with input from photometry), the soliton contribution to the gravitational potential requires a single free parameter in the fit. This parameter can be chosen to be, e.g., the soliton mass. For example, stellar kinematics in the MW could provide a testing ground for ULDM up to $m \sim 10^{-19} \mathrm{eV}$ [23]. To this end, implementing the soliton in a self-consistent manner would be crucial, and we expect that our tool would be useful.

Second, we revisit the analysis of Ref. [23] of baryonic effects in SPARC galaxies. As noted in Ref. [23], the soliton-halo relation predicted by DM-only numerical simulations strongly overpredicts the circular velocity in the centers of dozens of galaxies if $m<10^{-21} \mathrm{eV}$. In many cases, the predicted soliton mass in the central approximately $100 \mathrm{pc}$ of the galaxy exceeds the observationally allowed total mass (baryonic $+\mathrm{DM}$ ) in that region by factors of order 10. This large mass mismatch led the authors of Ref. [23] to expect that baryonic effects are unlikely to change the constraints. Here, focusing on two sample galaxies, we determine the soliton solution while accounting for the nonspherical baryonic mass distribution. When noting that the gas and stellar distributions are highly nonspherical, and when naively counting the mass outside of the soliton region, the total baryonic mass in both galaxies is comparable to or larger than the soliton mass. Nevertheless, in both cases, our analysis largely confirms the expectations of Ref. [23], showing that the baryonic mass external to the soliton region does not significantly affect the solution.

Some recent work in the literature investigated nonspherical distributions of condensed dark matter $[49,50]$. Reference [49] considered a nonspherical parametrization of ULDM halos and solitons and applied it to a Jeans analysis of dwarf spheroidal galaxies. Differently than our work here, Ref. [49] did not base its parametrization of the ULDM core on a solution of the equations of motion $(\mathrm{EOM})$. In our view, one of the main points of beauty in the discussion of ULDM in galaxies is that numerical simulations-with and without stars-actually do consistently show soliton solutions that satisfy the EOM. ${ }^{5}$ The tools we present here solve the EOM and find the selfconsistent soliton, allowing us to refine the analysis of Ref. [49]. Reference [50] looked for disk configurations of self-interacting condensed dark matter. However, while Ref. [50] looked for nonspherical configurations, at no point do they solve the EOM. Instead, they restrict the solution to certain disklike geometries and define and solve a modified one-dimensional system.

The outline of this paper is as follows. In Sec. II, we recall the ULDM equations of motion that define the soliton solution in the presence of an external (nondynamical) background gravitational potential. The standard onedimensional shooting method, that can be used to solve the spherically symmetric problem, becomes impractical (in general) once the background potential is not spherically symmetric because it requires a discrete infinity of shooting variables. ${ }^{6}$ A simple numerical recipe to solve this problem, assuming an axisymmetric background potential, is detailed in the Appendix A. While we do not pursue this here, extending the algorithm to full three dimensionality is straightforward.

In Sec. II A, we discuss the soliton-host halo mass relation found in DM-only numerical simulations $[9,10,32]$. Reference [23] shows that this relation is equivalent to the statement that the specific energy (total energy per unit mass) of the host halo is equal to the specific energy of a self-gravitating soliton. Here, we point out that a more physical representation of the soliton-halo relation is obtained by equating the kinetic-rather than total-energy per unit mass of the soliton and the halo. This distinction is unimportant for DM-only simulations of massive halos but becomes relevant once a background potential is introduced.

In Sec. III, we take the MW as an illustrative example of a system where the potential due to baryons (mostly stars in this case) cannot be neglected in assessing the soliton properties. The analysis demonstrates the use of our numerical tool but is not intended to provide constraints on ULDM; that would require a more comprehensive treatment that we postpone to future work.

In Sec. IV, we consider two sample LSB galaxies from the SPARC database. For these galaxies, we reconstruct the circular velocity decomposition presented in the SPARC database using photometric data, reproducing the SPARC analysis. The baryonic mass models (stars + gas) derived in this way are used as input for the numerical nonspherical soliton solution, allowing us to revisit in detail the earlier rough estimates of Ref. [23]. We show that the total energy

\footnotetext{
${ }^{5}$ See Secs. III and V. A in Ref. [23].

${ }^{6}$ If the problem is axisymmetric, for example, then one independent shooting variable is needed for every azimuthal Legendre $l$-mode.
} 
per unit mass, $E / M$, of the soliton is modified by the baryonic potential of these galaxies. However, the bulk of this effect is unphysical; it comes from a nondynamical shift of the energy due to an external gravitational potential that is mostly constant throughout the relevant region of the galaxy. This is supported by the fact that-as we showthe specific kinetic energy, $K / M$, is essentially unaffected both for the soliton and the halo.

In Sec. V, we summarize our results.

A number of technical details are postponed to Appendixes. As mentioned above, Appendix A describes the nonspherical soliton-finding algorithm. Appendix A 1 specifies the steps required to implement a black hole in the code. In Appendix B, we discuss tests of the algorithm and show evidence that the solutions we find are indeed ground-state solutions. In Appendix C, we recall a convenient formula converting an axisymmetric mass distribution into the gravitational potential induced by it. In Appendix D, we collect a useful auxiliary parametrization for galactic disks that we have found useful in modeling SPARC galaxies. Appendix E explains our reconstruction of the neutral gas distribution in UGC01281. Finally, our results in the main text are presented-for concretenessassuming a ULDM particle mass of $m=10^{-22} \mathrm{eV}$; in Appendix F, we show relevant results for $m=10^{-21} \mathrm{eV}$.

\section{SOLITONS IN A NONSPHERICAL BACKGROUND}

We consider a real, massive, free ${ }^{7}$ scalar field $\phi$ satisfying the Klein-Gordon equation of motion and minimally coupled to gravity. In the nonrelativistic regime, it is convenient to decompose $\phi$ as

$$
\phi(x, t)=\frac{1}{\sqrt{2} m} e^{-i m t} \psi(x, t)+\text { c.c. },
$$

with complex field $\psi$ that varies slowly in space and time and satisfies the Schrödinger-Poisson equations (SPEs) [53]

$$
\begin{gathered}
i \partial_{t} \psi=-\frac{1}{2 m} \nabla^{2} \psi+m\left(\Phi+\Phi_{b}\right) \psi, \\
\nabla^{2} \Phi=4 \pi G|\psi|^{2} .
\end{gathered}
$$

In Eq. (2), we include an external contribution to the gravitational potential, given by $\Phi_{b}$. We consider $\Phi_{b}$ as the effect of a distribution of baryonic mass. Our working assumption is that $\Phi_{b}$ should be constrained by external information such as photometry and microlensing measurements.

We look for a quasistationary phase-coherent solution for the ULDM, described by the ansatz

\footnotetext{
${ }^{7}$ Analyses of interacting fields can be found in, e.g., Refs. [7,8,51,52].
}

$$
\psi(x, t)=\left(\frac{m M_{p l}}{\sqrt{4 \pi}}\right) e^{-i \gamma m t} \chi(x),
$$

where $M_{p l}=1 / \sqrt{G}$. The parameter $\gamma$ is an eigenvalue of the SPE subject to the bound-state boundary conditions that we describe below.

We rescale the spatial coordinate,

$$
x \rightarrow m x,
$$

keeping this convention throughout the rest of the paper. Then, in terms of the dimensionless $\chi$ and $x$, the SPE are

$$
\begin{gathered}
\nabla^{2} \chi=2\left(\Phi+\Phi_{b}-\gamma\right) \chi, \\
\nabla^{2} \Phi=\chi^{2} .
\end{gathered}
$$

We assume cylindrical symmetry and parity symmetry $\left(x_{3}=z \rightarrow-z\right)$ and define the radial coordinate in the plane $R=\sqrt{x_{1}^{2}+x_{2}^{2}}$. At $\sqrt{R^{2}+z^{2}} \rightarrow \infty$, the potentials $\Phi$ and $\Phi_{b}$ are assumed to decay $\propto 1 / \sqrt{R^{2}+z^{2}}$, implying that $\chi$ decays approximately exponentially $\propto e^{-\sqrt{2|\gamma|\left(R^{2}+z^{2}\right)}}$. A given value of $\chi$ at the origin, specified by

$$
\chi(R=0, z=0)=\lambda^{2}
$$

with $\lambda$ a real positive number, fixes the minimal energy solution of Eqs. (6) and (7) consistent with the boundary conditions.

In the case of vanishing $\Phi_{b}$, solutions of Eqs. (6) and (7) admit a scaling symmetry, the orbit of which can be parametrized by $\lambda$. This scaling symmetry is, in general, broken by $\Phi_{b} \neq 0$. It remains true, however, that varying the value of $\lambda$ in Eq. (8) generates the continuous family of solutions of Eqs. (6) and (7). Thus, $\lambda$ remains a useful tool to parameterize the mass, energy, and any other property of the solution. For reference, the self-gravitating soliton (found for $\Phi_{b}=0$ ) satisfies $M \approx$ $2.06 \lambda \frac{M_{p l}^{2}}{m} \approx 2.8 \times 10^{12} \lambda\left(\frac{m}{10^{-22} \mathrm{eV}}\right)^{-1} M_{\odot}$ and $E / M \approx-0.23 \lambda^{2} \approx$ $-0.054\left(\frac{M}{M_{p l}^{2} / m}\right)^{2}$. When baryons induce $\Phi_{b} \neq 0$, these relations are modified in a way that we will discuss below.

We have developed a numerical relaxation method to find the ground-state soliton solution for any axisymmetric background potential satisfying the boundary conditions described below Eq. (7). The algorithm is described in Appendix A and is one of the main results of this paper. We discuss some theoretical aspects of the solutions in Appendix B: first, the evidence for (but difficulty of rigorously proving) that the solution is indeed the ground state and, second, the issue of stability against small perturbations.

In the next subsection, we clarify some issues related to the soliton-host halo relation found in DM-only numerical simulations. Then, in the following sections, we illustrate the use of the numerical tool of Appendix A by analyzing 
the baryonic effects on the predicted ULDM soliton in the Milky Way and in two disk galaxies from the SPARC database.

\section{A. Soliton-halo relation: $E / M$ vs $K / M$}

We can compute the soliton mass $M$ and energy $E$ (recall that $x$ is measured in units of $1 / m$ ),

$$
\begin{aligned}
M & \approx 10^{11} M_{\odot}\left(\frac{m}{10^{-22} \mathrm{eV}}\right)^{-1} \int d^{3} x \chi^{2}, \\
E \approx & 10^{11} M_{\odot}\left(\frac{m}{10^{-22} \mathrm{eV}}\right)^{-1} \\
& \times \int d^{3} x\left(\frac{1}{2}(\nabla \chi)^{2}+\left(\frac{\Phi}{2}+\Phi_{b}\right) \chi^{2}\right) .
\end{aligned}
$$

It is useful to separate the total energy into kinetic energy + potential energy,

$$
E=K+P,
$$

where $K$ comes from the gradient term and $P$ comes from the $\Phi / 2+\Phi_{b}$ term in Eq. (10). For a self-gravitating system in virial equilibrium, $P=-2 K$ and $E=-K$. This applies to the self-gravitating soliton obtained for $\Phi_{b}=0$. When we turn on a background potential, the soliton ceases to be self-gravitating, so that $E \neq-K$ for $\Phi_{b} \neq 0$.

Reference [23] showed that the empirical soliton-host halo relation found in the DM-only numerical simulations of Ref. [10] is equivalent to the statement

$$
\left.\frac{E}{M}\right|_{\text {soliton }}=\left.\frac{E}{M}\right|_{\text {halo }} .
$$

Note that on the lhs of Eq. (12) $\left.\frac{E}{M}\right|_{\text {soliton }}$ is defined for the self-gravitating soliton without including the gravitational potential induced by the large-scale halo. The halo gravitational potential $\Phi_{h}$ is approximately constant in the halo inner region where the soliton occurs and can be estimated as $\left.\Phi_{h} \sim 10 \frac{E}{M}\right|_{\text {halo }}$, up to $\mathcal{O}(1)$ corrections depending on the detailed shape of the halo [23]. If we were to include the correction to the soliton energy due to this constant background potential, it would change: $\left.\left.\frac{E}{M}\right|_{\text {soliton }} \rightarrow \frac{E}{M}\right|_{\text {soliton }}+$ $\Phi_{h}$. This discussion suggests that the soliton-host halo relation is better expressed using kinetic energy, rather than total energy:

$$
\left.\frac{K}{M}\right|_{\text {soliton }}=\left.\frac{K}{M}\right|_{\text {halo }} .
$$

Because $\Phi_{h}$ is approximately constant over the region where the soliton is supported, the soliton shape is not distorted, and its kinetic energy is not modified from its value for the self-gravitating solution. This means that for massive halos in DM-only simulations Eqs. (13) and (12) are indistinguishable.
Equations (13) and (12) become distinguishable when we turn on $\Phi_{b} \neq 0$, with a nontrivial spatial profile such that $\Phi_{b}$ is not constant throughout the large-scale halo.

\section{APPLICATION: THE MILKY WAY}

We now consider soliton solutions in the background of a gravitational potential $\Phi_{b}$, chosen to roughly mimic the inner region of the MW. Our goal is to illustrate the approximate size of the baryonic effects on the soliton and not to characterize these effects in full; a detailed, accurate, and precise modeling of the inner MW stellar and gas mass distributions is challenging and is postponed to future work. For concreteness, throughout this section, we set $m=10^{-22} \mathrm{eV}$.

The dominant contributions to the stellar mass profile of the MW inner few hundred parsecs were described in the photometric analysis of Launhardt et al. [54] as a spherical nuclear stellar cluster (NSC) and a nuclear stellar disk (NSD), composing together the nuclear bulge (NB).

In addition to the stellar components, dynamics in the central approximately $1 \mathrm{pc}$ is dominated by a supermassive black hole $(\mathrm{SMBH})$ with mass $M_{\mathrm{BH}} \approx 4 \times 10^{6} M_{\odot}$. Here, we omit the SMBH contribution, which was studied in Ref. [23] and shown to have a negligible impact on the soliton for $m \lesssim 10^{-20} \mathrm{eV}$. We note that the numerical code in Appendix $\mathrm{A}$ is capable of handling the $\mathrm{SMBH}$ contribution via the procedure described in Appendix A 1. A gas torus at a scale radius of approximately 100 pc contributes approximately $2 \times 10^{7} \mathrm{M}_{\odot}$. For simplicity, the gas is also neglected here in comparison to the stellar components.

The NSC density profile was modeled as

$$
\rho_{\mathrm{NSC}}(r)=\frac{\bar{\rho}_{\mathrm{NSC}}}{1+\left(\frac{r}{0.22}\right)^{n_{\mathrm{NSC}}}} \theta(200-r),
$$

where $r=\sqrt{R^{2}+z^{2}}$ is stated in parsecs. $\bar{\rho}_{\mathrm{NSC}}=3.3 \times$ $10^{6} M_{\odot} / \mathrm{pc}^{3}$ for $r<r_{0}$, and $\bar{\rho}_{\mathrm{NSC}}=9.0 \times 10^{7} M_{\odot} / \mathrm{pc}^{3}$ for $r \geq r_{0}$, with $r_{0}=6 \mathrm{pc}$. The index $n_{\mathrm{NSC}}=2$ for $r<r_{0}$, and $n_{\mathrm{NSC}}=3$ for $r \geq r_{0}$ (keeping the profile continuous at $r_{0}$ ). With these parameters, we have ${ }^{8} M_{\mathrm{NSC}} \simeq 5.3 \times 10^{7} \mathrm{M}_{\odot}$.

We parametrize the NSD stellar mass density as follows,

$\rho_{\mathrm{NSD}}(R, z)=\frac{\bar{\rho}_{\mathrm{NSD}}}{1+\left(\frac{R}{250}\right)^{14}}\left(1-\tanh ^{4}\left(\frac{R}{140}\right)\right) e^{-\frac{|k|}{15}}$,

where $\bar{\rho}_{\text {NSD }}=330 M_{\odot} / \mathrm{pc}^{3}$ and where $z$ and $R$ are stated in parsecs. This parametrization approximately reproduces the near infra-red stellar volume emissivity model derived in Ref. [54] and yields a NSD mass $M_{\mathrm{NSD}} \simeq 10^{9} \mathrm{M}_{\odot}$,

\footnotetext{
${ }^{8}$ This NSC mass is larger than that quoted in Ref. [54] by a factor of approximately 1.8. We are not sure of the reason for this mismatch, but it does not have an important effect on our results.
} 
consistent within the uncertainty with the value of $(1.4 \pm 0.6) \times 10^{9} \mathrm{M}_{\odot}$ quoted by Ref. [54].

A kinematic detection supporting the disklike morphology of the NSD was given in Ref. [55], and the mass and approximate scale estimates are consistent with the dynamical modeling of Ref. [56] and with microlensing analyses [57] that probe the outer boundary of the NSD region.

In what follows, we define $\Upsilon_{L} \equiv \Upsilon / \Upsilon_{\text {Launhardt }}$ as the mass-to-light ratio of the stellar distribution compared to the one used in Ref. [54]. We vary $\Upsilon_{L}$ to explore the consequences of different total stellar mass in the NB region.

In Fig. 1, we plot the soliton mass vs $\lambda$, which allows us to access different solutions. For $\lambda \gtrsim 10^{-3}$, we retrieve the self-gravitating soliton result, shown by the dashed line. For smaller $\lambda$, we find $M \propto \lambda^{4}$ [23]. ${ }^{9}$ Figure 1 can be compared to Fig. 16 in Ref. [23], which considered a spherically averaged approximation to the same stellar mass model. It shows an $\mathcal{O}(1)$ difference in the $M$ vs $\lambda$ relation in the phenomenologically interesting range $\lambda \sim 10^{-4}-10^{-3}$.

In Fig. 2, we study the deformation in the soliton shape caused by the stellar mass distribution, at fixed soliton mass $M \approx 1.35 \times 10^{9} \mathrm{M}_{\odot}$ predicted by DM-only numerical simulations for a halo mass $M_{h}=10^{12} \mathrm{M}_{\odot}$. The contour lines show the soliton mass density normalized to a reference value of $23.6 \mathrm{M}_{\odot} / \mathrm{pc}^{3}$. Solid lines show the result for the self-gravitating soliton, and dashed lines show the result obtained when $\Phi_{b}$ is included in the SPE. In Fig. 3, we plot the density profile of the deformed soliton from Fig. 2 on the plane of the disk $(z=0$, dashed line) and along the $z$ axis $(R=0$, dot dashed). The solid line shows the density profile of the self-gravitating soliton. The dotted line shows the density profile of the soliton when the baryonic potential is replaced by a radially averaged version of the potential. ${ }^{10}$

It is instructive to consider the observable (in principle) soliton-induced effective circular velocity,

$$
v_{\text {eff }}(x)=\sqrt{x \cdot \nabla \Phi}
$$

In Fig. 4, we plot $v_{\text {eff }}$, analogously to Fig. 3. The dashed line is $v_{\text {eff }}$ on the plane of the disk. The dot-dashed line is $v_{\text {eff }}$ transverse to the disk on the $z$ axis. For comparison, we also plot $v_{\text {eff }}$ computed for a self-gravitating soliton with the same mass (solid blue). The main effect of the

\footnotetext{
${ }^{9}$ This can be understood as follows. For small $\lambda$, the external potential dominates, and the SPEs reduce to $\nabla^{2} \chi \approx 2\left(\Phi_{b}-\gamma\right) \chi$. Since this equation is homogeneous and linear in $\chi$, the normalization at $x=0$ is a multiplicative factor, and $M \propto \int d^{3} x \chi^{2} \propto \lambda^{4}$.

${ }^{10}$ Specifically, we define the spherical rearrangement via $M(r)=\int_{0}^{r} \rho(\mathbf{x}) d^{3} x, \phi(r)=-\int_{r}^{\infty} d r^{\prime} G M\left(r^{\prime}\right) /\left(r^{\prime}\right)^{2}$.
}

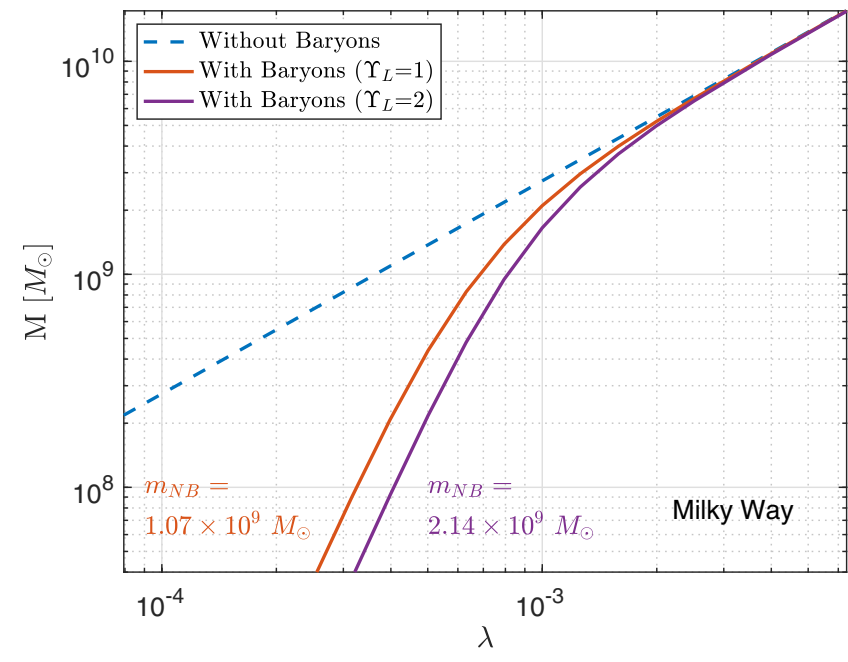

FIG. 1. Soliton $M-\lambda$ relation in the stellar-induced background gravitational potential of the inner MW. For a halo mass $M_{h}=10^{12} \mathrm{M}_{\odot}$, the soliton-host halo relation found in DM-only numerical simulations predicts $\lambda=4.9 \times 10^{-4}$. The ULDM particle mass is $m=10^{-22} \mathrm{eV} . \Upsilon_{L}$ is defined in the text.

background stellar potential is to contract the solitoninduced peak velocity deeper into the inner halo, enhancing the peak velocity; this is an $\mathcal{O}(1)$ effect that cannot be ignored in realistic modeling of kinematic data. The deviation from radial symmetry is, however, small: a simplified treatment taking as input a radially averaged baryonic mass distribution could suffice for practical purposes. For comparison, the result of such a procedure is plotted in the dotted line in Fig. 4.

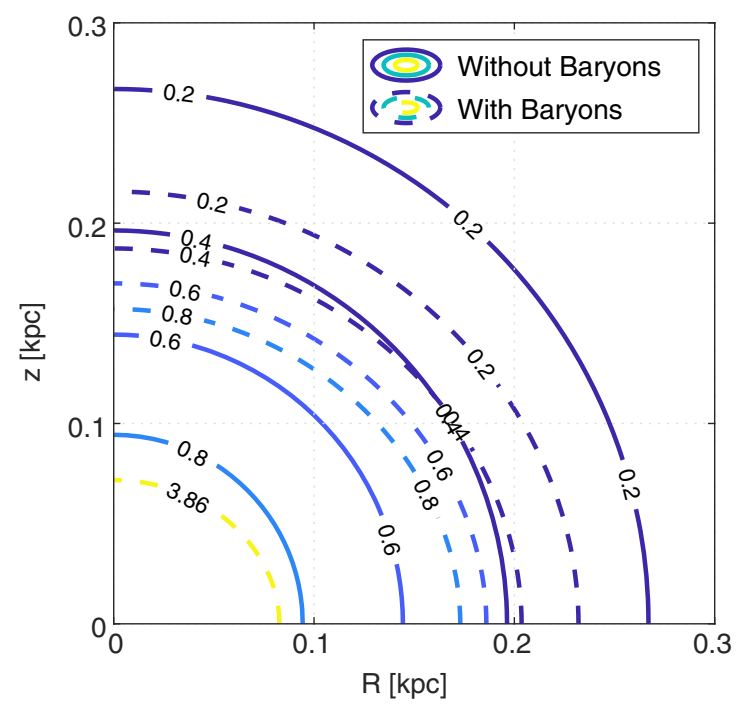

FIG. 2. Mass density contours of a soliton in the inner MW. The density is normalized to a reference value of $23.6 \mathrm{M}_{\odot} / \mathrm{pc}^{3}$. We set $m=10^{-22} \mathrm{eV}$ in the plot. The soliton mass is fixed at $M \approx 1.35 \times 10^{9} \mathrm{M}_{\odot}$. Solid lines show the result for the selfgravitating soliton, and dashed lines show the result when $\Phi_{b}$ is included in the SPE. 


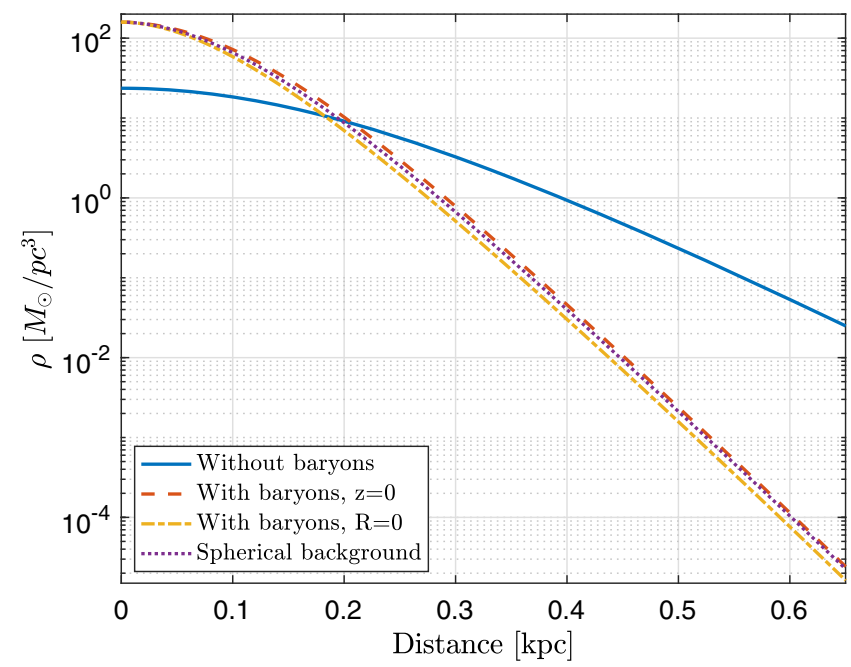

FIG. 3. Mass density of solitons corresponding to the inner MW. We set $m=10^{-22} \mathrm{eV}$ in the plot. The soliton mass is fixed at $M \approx 1.35 \times 10^{9} \mathrm{M}_{\odot}$. The solid line shows the result for the self-gravitating soliton, and the dashed lines show the results when $\Phi_{b}$ is included in the SPE. The dotted line shows the result when the NSD is replaced by a spherical rearrangement of the same mass.

In the top (bottom) panel of Fig. 5, we plot the total energy (kinetic energy) per unit mass as a function of soliton mass $M$. For $M \gtrsim 10^{10} M_{\odot}$, the self-gravitating soliton result is retrieved. For small $M$, we find that $E / M$ and $K / M$ approach constant values. The reason for this

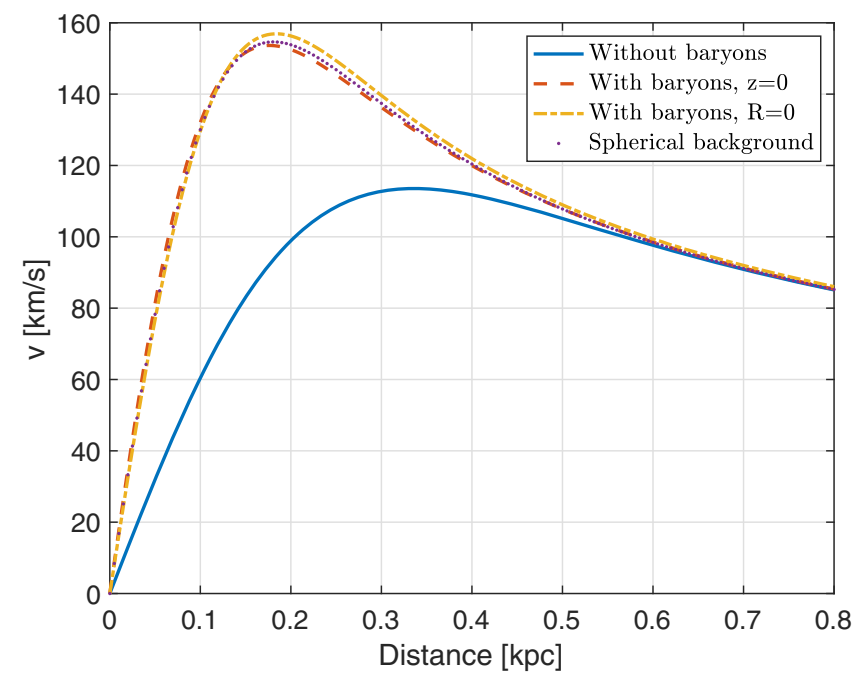

FIG. 4. Effective circular velocity induced by a MW soliton. We set $m=10^{-22} \mathrm{eV}$ in the plot and fix the soliton mass at $M \approx 1.35 \times 10^{9} \mathrm{M}_{\odot}$, predicted by DM-only numerical simulations for a halo mass $M_{h}=10^{12} \mathrm{M}_{\odot}$. The solid line shows the result for the self-gravitating soliton, and the dashed lines show the result when $\Phi_{b}$ is included in the SPE. The dotted line shows the result when the NSD is replaced by a spherical rearrangement of the same mass. scaling follows along the same lines of footnote 9, which shows that at small $\lambda$, when the background potential dominates, $M, E$, and $K$ all scale as $\propto \lambda^{4}$ leading to constant $E / M$ and $K / M$.

For a halo mass $M_{h}=10^{12} M_{\odot}$, the soliton-host halo relation found in DM-only numerical simulations of Refs. [9,10] [summarized by Eq. (13)] predicts $K / M \approx$ $5.5 \times 10^{-8}$ [23], shown by the black dot-dashed line. The shaded band denotes a factor of 2 spread around this prediction, motivated by the halo-to-halo spread seen in the simulations.

Figure 5 shows that, because of the stellar-induced background potential, $K / M$ for an actual soliton solution in this background is significantly deformed. This means that baryonic effects are likely to significantly modify the
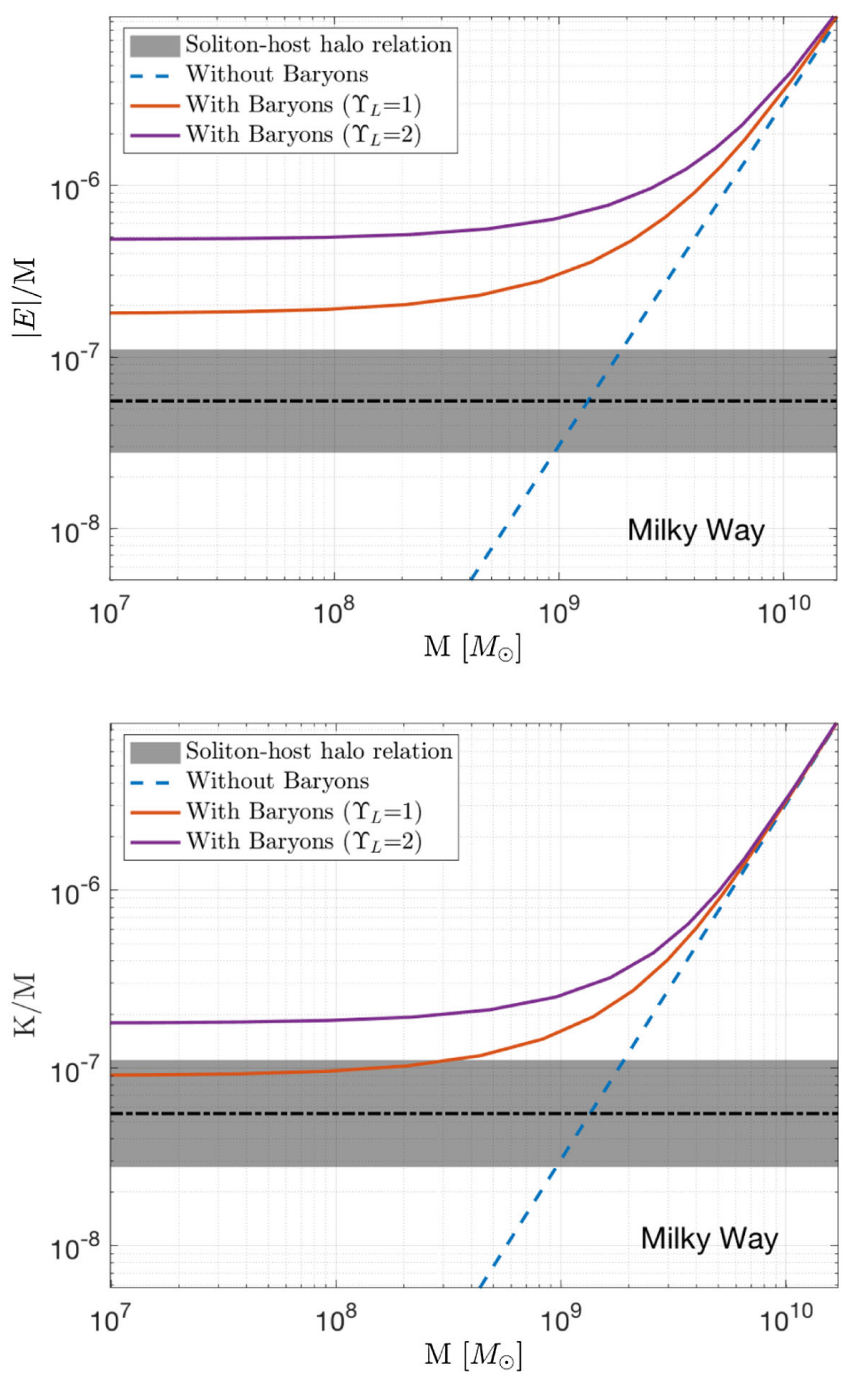

FIG. 5. Specific energy $|E| / M$ (top) and specific kinetic energy $K / M$ (bottom) for a soliton in the MW. For a halo mass $M_{h}=10^{12} M_{\odot}$, the Soliton-Host Halo Relation found in DMonly numerical simulations predicts $|E| / M=K / M \approx 5.5 \times 10^{-8}$ [23], shown by the black dot-dashed line with a shaded band denoting a factor of 2 spread (see the text for more details). 
soliton properties, and the soliton-halo expectation from DM-only numerical simulations cannot be taken at face value. A consistent way to constrain (or possibly detect) an ULDM soliton in the MW, would be by a combined analysis of kinematical modeling and photometry, where the stellar potential constrained by photometry is used to self-consistently calculate the soliton shape and where the soliton mass is taken as a free parameter.

Reference [58] argued for dynamical evidence in favor of an ULDM soliton in the MW, with $m \approx 10^{-22} \mathrm{eV}$ and $M \approx$ $10^{9} M_{\odot}$ in tantalizing agreement with the expectations of DM-only numerical simulations. The dynamical evidence for a dense central mass component is consistent with earlier studies [23,54-56]. Unfortunately, as we reviewed here and in Ref. [23] (see Sec. V. B there), there is room for and photometric evidence of about $10^{9} M_{\odot}$ in stars within the approximately $200 \mathrm{pc}$ would-be soliton region [54]. Thus, the central mass component could well be due to ordinary baryonic matter. Other systems, such as wellresolved LSB galaxies, offer much cleaner laboratories in which to look for ULDM solitons. We turn to such systems in the next section.

\section{APPLICATION: LOW-SURFACE BRIGHTNESS SPARC GALAXIES}

Our second discussion of nonspherical solitons involves two LSB disk galaxies from the SPARC database [31]: UGC01281 and F571-8. We choose these galaxies as representative examples of a larger sample including dozens of well-resolved LSB galaxies. For concreteness, throughout this section, we set $m=10^{-22} \mathrm{eV}$. Results for $m=10^{-21} \mathrm{eV}$ are collected in Appendix F.

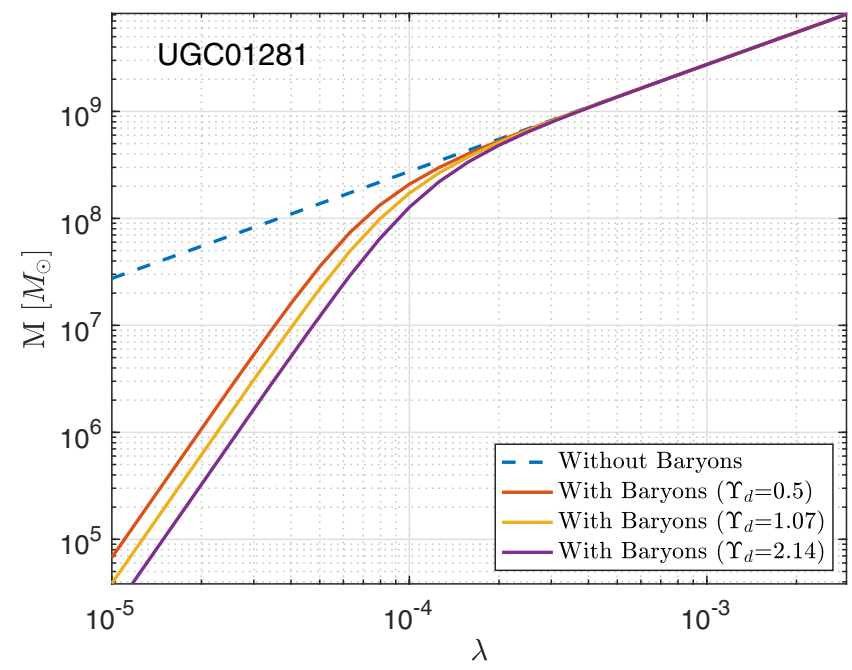

FIG. 6. Soliton $\mathrm{M}-\lambda$ relation in the baryonic-induced background gravitational potential of UGC01281. The Soliton-Host Halo Relation found in DM-only numerical simulations predicts $\lambda=2.2 \times 10^{-4}$. The ULDM particle mass is $m=10^{-22} \mathrm{eV}$.
The baryonic mass contributions in SPARC galaxies is divided into a spherical bulge component and axisymmetric disk and gas components. The stellar mass distribution is calibrated to match surface-brightness data from Spitzer. The computation of the gravitational potential due to the disk is detailed in Appendix D. We focus here on galaxies that are consistent with negligible bulge.

The gas mass distribution for UGC01281 (not relevant for F571-8) is calibrated to approximately match the HI surface brightness data reported in Ref. [59], normalizing to the total gas mass reported in Ref. [60]. We provide details on the gas fitting procedure in Appendix E.

In our computation, we fix the total gas mass to match the total mass inferred from the photometry and vary the stellar mass-to-light ratio of the disk from $\Upsilon_{d}=0$ up to larger values that saturate the observed kinematic velocity [61].
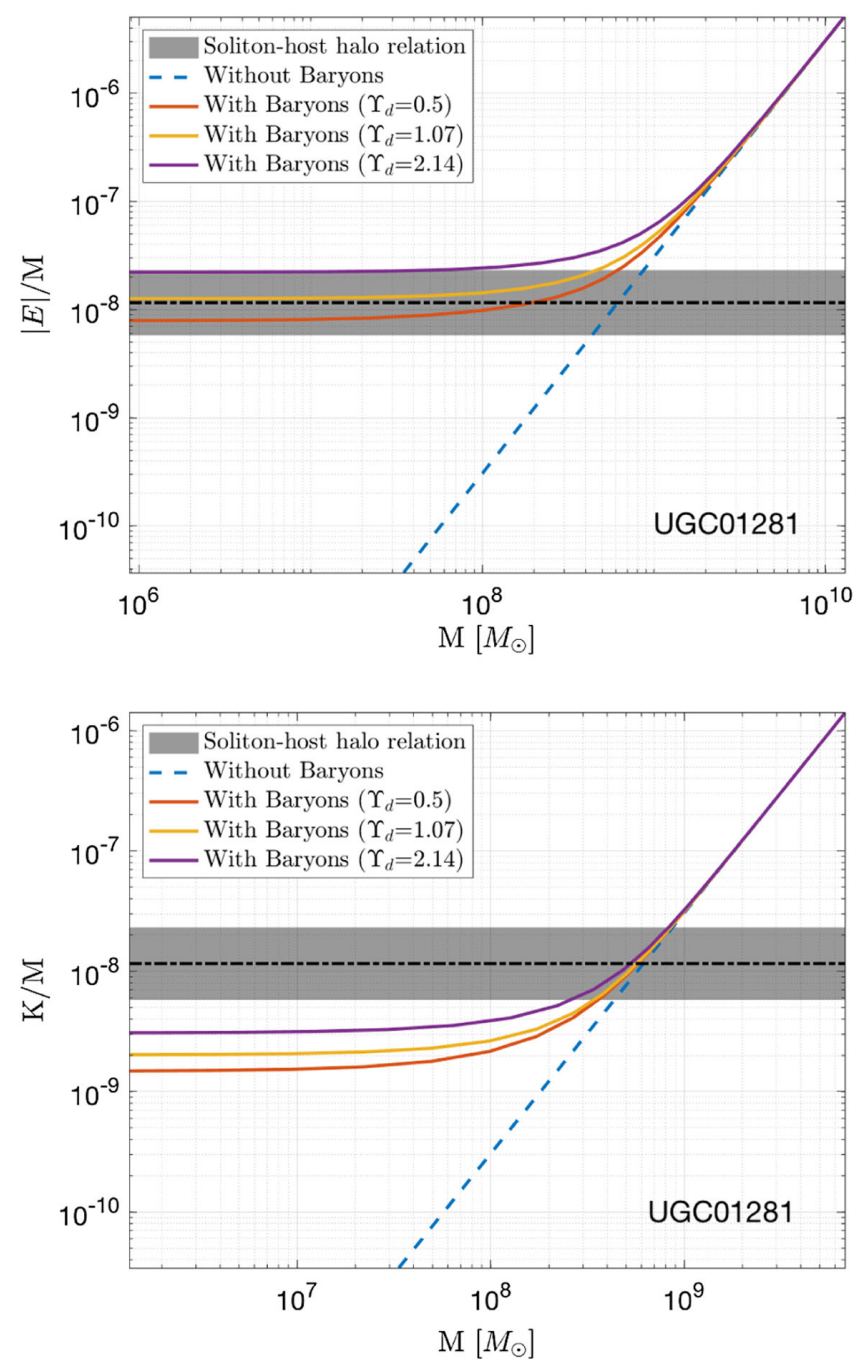

FIG. 7. Specific energy $|E| / M$ (top) and specific kinetic energy $K / M$ (bottom) for a soliton in UGC01281. The Soliton-Host Halo Relation found in DM-only numerical simulations predicts $|E| / M=K / M \approx 10^{-8}$ [23], shown by the black dashed line with a shaded band denoting a factor of 2 spread. 
In Fig. 6, we plot the $M-\lambda$ relation for a soliton in UGC01281. In the top (bottom) panel of Fig. 7, we plot the total energy (kinetic energy) per unit mass vs $M$. The dashed black line denotes the soliton-halo prediction of DM-only numerical simulations. The shaded band shows a factor of 2 spread around this prediction.

Inspecting Fig. 7, we see that in the neighborhood of $E / M$ values that conform to the DM-only simulation prediction the actual $E / M$ for a soliton in UGC01281 is significantly shifted compared to the self-gravitating solution. However, the effect on $K / M$ is much less pronounced; the soliton shape is essentially unaffected.

We can also estimate the baryonic effect on the dynamics of the large-scale halo. To do this, we can compare the observed kinematic velocity at large distances $(r \sim 5 \mathrm{kpc}$ in this example) with the contribution to the velocity that can be attributed to the baryons. The velocity decomposition is shown in the top panel of Fig. 10 (discussed in more detail at the end of this section). We find $v_{\text {baryons }}^{2} / v_{\text {obs }}^{2} \sim 0.26$ ( 0.39), when adopting $\Upsilon_{d}=1.07 \quad\left(\Upsilon_{d}=2.14\right)$. This means that the baryonic potential distorts the ULDM large-scale halo $K / M$ by no more than $40 \%$.

The next galaxy we consider is F571-8. Soliton properties for this galaxy are presented in Figs. 8 and 9. Here, for simplicity, we ignore the (negligible) gas contribution in computing the soliton. Again, $K / M$ for a soliton in F571-8 is unaffected by baryons in the parameter region expected from DM-only simulations. The case of F571-8 is even clearer than UGC01281 because the baryonic effect on the dynamics of the large-scale halo, as seen by inspecting the rotation curve decomposition (bottom panel of Fig. 10), is not larger than $\sim 5 \%$.

In the top (bottom) panel of Fig. 10, we show the rotation curve decomposition of UGC01281 and F571-8, as found in the SPARC database. The contributions due to soliton solutions with different values of $\lambda$ (indicated in the plot) are overlaid in red, blue, and black. The solitons are

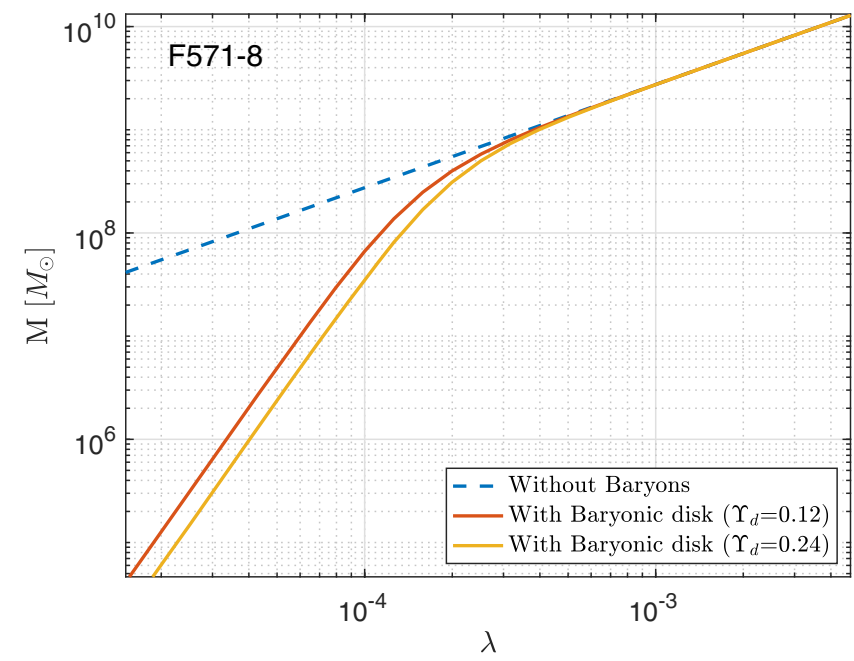

FIG. 8. Same as Fig. 6, but done for F571-8. computed assuming different values of the disk stellar mass-to-light ratio $\Upsilon_{d}$, listed in the caption. The central value of $\lambda$ (in blue) corresponds to the prediction of DM-only numerical simulations. For these predicted solitons, the baryonic potential makes a negligible impact on the soliton shape, regardless of the value of $\Upsilon_{d}$ in both galaxies.

We conclude that if Eq. (13) correctly captures the soliton-halo relation of the simulations then UGC01281 and F571-8 are clean systems in which to constrain the ULDM model, in the sense that the baryonic contribution to the gravitational potential is not important for neither the large-scale halo nor the central soliton. These conclusions stay unchanged when we consider more massive ULDM with $m=10^{-21} \mathrm{eV}$ (see Appendix F). Dozens of other comparably clean systems exist in the SPARC database. The constraints derived in Ref. [23] should therefore apply, and ULDM with $m<10^{-21} \mathrm{eV}$ is in tension with the data.
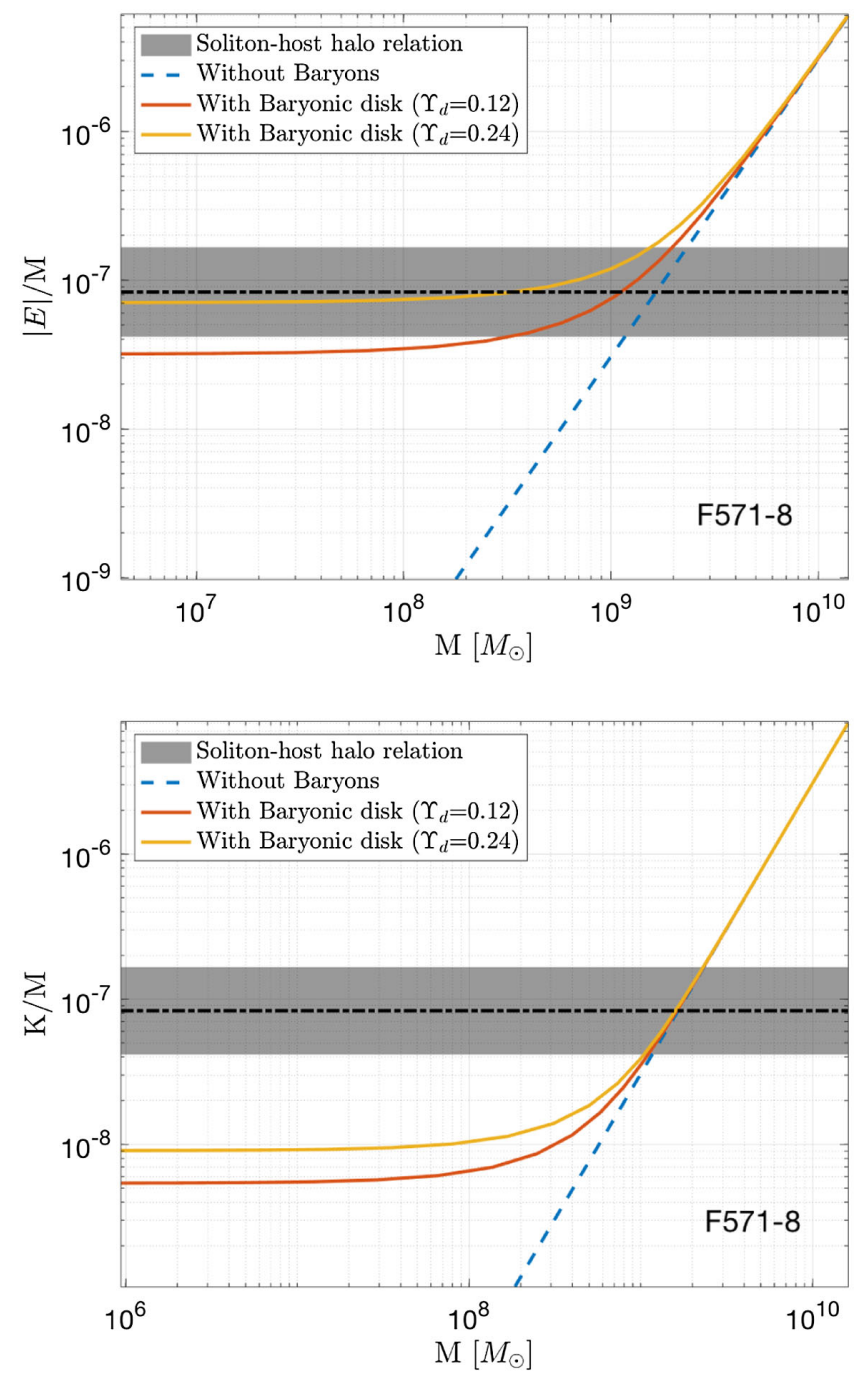

FIG. 9. Same as Fig. 7, but done for F571-8. 

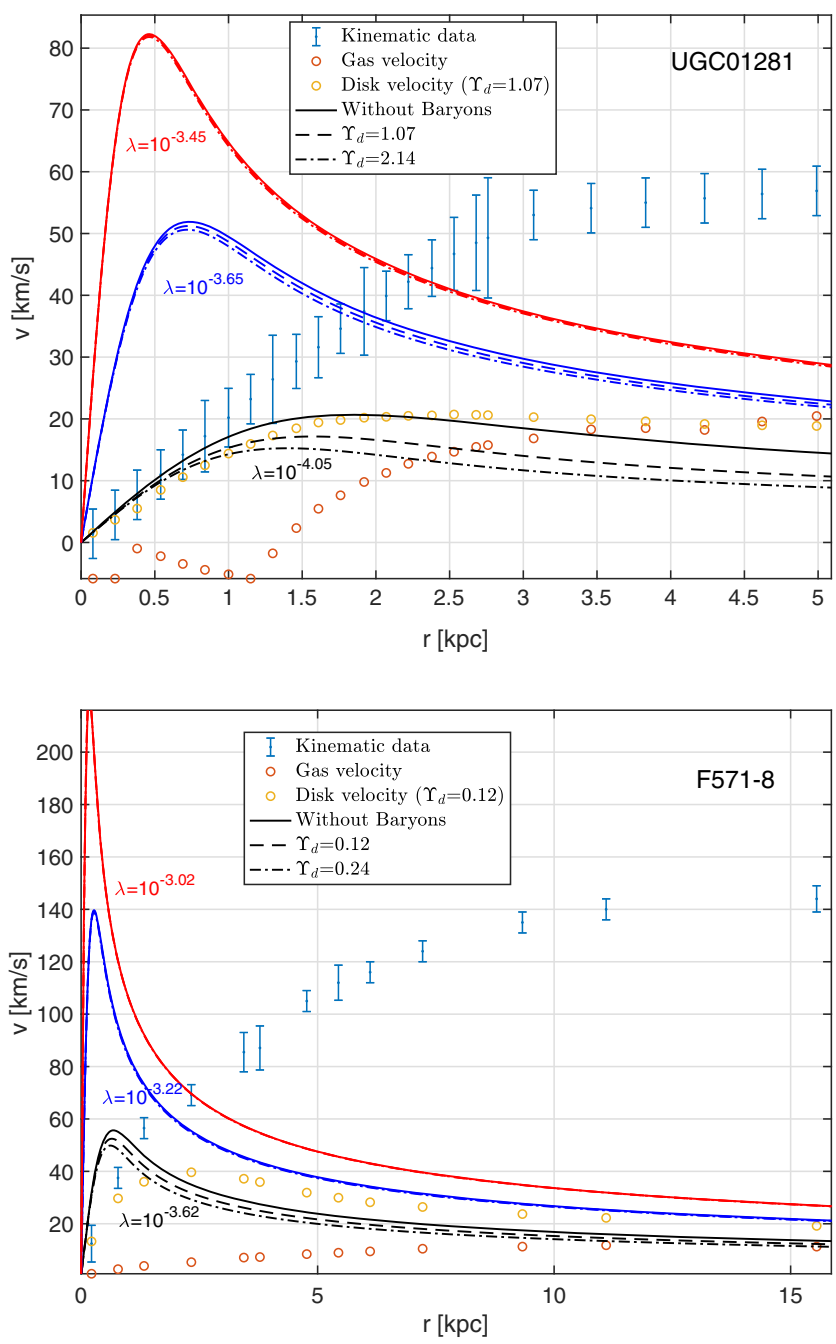

FIG. 10. Top: Rotation curve SPARC data of UGC01281, overlaid with soliton solutions assuming background baryonicinduced potential parametrized by stellar disk mass-to-light ratio $\Upsilon_{d}$ augmented by neutral gas mass distribution consistent with the observed HI brightness measurements. The highest $\Upsilon_{d}$ is chosen to saturate the error budget of the innermost kinematic velocity data points. The central value, $\lambda=10^{-3.65}$ (blue), is based on the DM-only numerical simulation prediction. Bottom: Same for F571-8 (for this galaxy, the gas contribution is neglected in the soliton computation). The DM-only simulations predict $\lambda=10^{-3.22}$.

\section{CONCLUSIONS}

An ultralight bosonic field oscillating around a minimum of its potential can play the role of dark matter. On scales of order the effective de Broglie wavelength, wave mechanics dictates the dynamics of this ultralight dark matter, opening potential avenues to constrain (or detect) ULDM in various astrophysical and cosmological systems.

Stellar and gas kinematics of rotation-supported lowsurface brightness galaxies were used in Ref. [23] to derive the constraint $m \gtrsim 10^{-21} \mathrm{eV}$. This constraint relies on the validity of a soliton-host halo relation, found in DM-only numerical simulations. It is important to assess to what extent baryons could affect these results. For a nonspherical baryonic distribution, a new numerical tool was required in order to calculate the properties (shape, mass, and energetics) of the nonspherical soliton obtained in the presence of the baryonic-induced background gravitational potential. In this paper, we provided a simple algorithm (see Sec. II and Appendix A) that achieves this goal.

To illustrate the potential use of the nonspherical soliton solver, we estimated the impact of a Milky Way nuclear stellar disk on an ULDM soliton. Adopting a plausible parameterization of the stellar distribution, motivated by photometric measurements, we find that the NSD would distort the shape and energetics of an $m=10^{-22} \mathrm{eV} \mathrm{ULDM}$ soliton at the $\mathcal{O}(1)$ level. Thus, an attempt to constrain ULDM in the MW should self-consistently account for the gravitational effect of stars. While we did not enter such an analysis, the numerical tool we provided is an important step in this direction. Having said that, we note that, while the soliton can be compressed by an internal clump of stars, it is not easily deformed into nonspherical shape. In the MW example, the highly nonspherical nuclear stellar disk leads to a soliton that is significantly contracted but remains spherical to a good approximation. As a result, a spherical rearrangement of the stellar mass distribution (namely, replacing the disklike baryonic distribution by a radially averaged profile) would most likely be sufficient to calculate the soliton in a kinematical analysis.

Next, we revisited the SPARC galaxy analysis of Ref. [23]. Using two LSB galaxies as a concrete example, we modeled the baryonic potential consistent with photometric data and bracketed the possible impact on the shape and energetics of the predicted soliton. Our results reinforce the conclusions of Ref. [23], implying that baryons are not expected to change the constraints derived on ULDM based on rotationally supported LSB SPARC galaxies.

\section{ACKNOWLEDGMENTS}

We thank Ben Bar-Or and Scott Tremaine for discussions and Stacy McGaugh for clarifications regarding the SPARC database. K. B. is incumbent of the Dewey David Stone and Harry Levine career development chair. The work of K. B. and N. B. was supported by Grant No. 1937/ 12 from the I-CORE program of the Planning and Budgeting Committee and the Israel Science Foundation and by Grant No. 1507/16 from the Israel Science Foundation. The work of J.E. was supported by the Zuckerman STEM Leadership Program.

\section{APPENDIX A: NUMERICAL ALGORITHM FOR SOLITONS IN A NONSPHERICAL BACKGROUND POTENTIAL}

In what follows, we describe a numerical method to find the ground-state solution of Eqs. (6) and (7). This tool is 
one of the main results of our work; it is intended to be simple, fast, and robust enough so it can be used in detailed analyses of galactic kinematics with ULDM, in casessuch as the Milky Way Galaxy-where the baryonic contribution to the gravitational potential in the soliton region cannot be neglected.

We assume that the baryonic-induced gravitational potential is a direct input to the code. Often, an input in terms of the stellar and gas mass density could be more natural. Converting an axisymmetric mass distribution into its corresponding gravitational potential is a straightforward exercise that we recall in Appendix C.

We use an $N \times N$ discretized lattice with physical size $L \times L$ in the $R-z$ plane. The lattice spacing is $\delta=L /(N-1)$. The physical coordinate of each point (recall that distance is measured in units of $1 / \mathrm{m}$ ) is

$$
\left(R_{i}, z_{j}\right)=\left(\frac{i-1}{N-1} L, \frac{j-1}{N-1} L\right)
$$

The Laplacian in cylindrical coordinates is

$$
\nabla^{2} \Phi=\left(\frac{\partial^{2}}{\partial R^{2}}+\frac{1}{R} \frac{\partial}{\partial R}\right) \Phi+\frac{\partial^{2}}{\partial z^{2}} \Phi
$$

We discretize it:

$$
\begin{aligned}
& {\left[\left(\frac{\partial^{2}}{\partial R^{2}}+\frac{1}{R} \frac{\partial}{\partial R}\right) \Phi\right]_{i, j}} \\
& = \begin{cases}\frac{4\left(\Phi_{2, j}-\Phi_{1, j}\right)}{\delta^{2}} & (i=1) \\
\frac{\Phi_{i+1, j}-2 \Phi_{i, j}+\Phi_{i-1, j}}{\delta^{2}}+\frac{1}{R_{i}} \frac{\Phi_{i+1, j}-\Phi_{i-1, j}}{2 \delta} & (1<i<N)\end{cases} \\
& {\left[\frac{\partial^{2}}{\partial z^{2}} \Phi\right]_{i, j}= \begin{cases}\frac{2\left(\Phi_{i, 2}-\Phi_{i, 1}\right)}{\delta^{2}} & (j=1) \\
\frac{\Phi_{i, j+1}-2 \Phi_{i, j}+\Phi_{i, j-1}}{\delta^{2}} & (1<j<N) .\end{cases} }
\end{aligned}
$$

Note that we do not need to define $\nabla^{2} \Phi$ at $i=N$ nor $j=N$.

We start by initializing $\Phi$ as zero everywhere, assigning an initial test profile of $\chi$ that is conveniently chosen as some numerical approximation of the known self-gravitating solution; see, e.g., Ref. [11]. Throughout the calculation, we enforce

$$
\chi_{i, N}=\chi_{N, i}=0 .
$$

The discretized Eq. (7) is then solved iteratively using the successive over-relaxation (SOR) method (see, e.g., Chap. 19.5 in Ref. [62]). In each iteration of the program, $\Phi_{i, j}(i, j \neq 1, N)$ is improved by the SOR method as

$$
\begin{aligned}
\Phi_{i, j}^{\text {new }}= & \Phi_{i, j}^{\text {old }}+\frac{\omega_{\Phi} \delta^{2}}{4} \\
& \times\left(\frac{\Phi_{i+1, j}^{\text {old }}-2 \Phi_{i, j}^{\text {old }}+\Phi_{i, j-1}^{\text {new }}}{\delta^{2}}+\frac{\Phi_{i+1, j}^{\text {old }}-\Phi_{i-1, j}^{\text {new }}}{2 \delta R_{i}}\right. \\
& \left.+\frac{\Phi_{i, j+1}^{\text {old }}-2 \Phi_{i, j}^{\text {old }}+\Phi_{i, j-1}^{\text {new }}}{\delta^{2}}-\chi_{i, j}^{2}\right),
\end{aligned}
$$

where $\omega_{\Phi}$ is an auxiliary parameter ${ }^{11}$ that we set $\mathcal{O}(1)$. For $i=1$ and/or $j=1$, the rhs of Eq. (A6) should be modified according to Eqs. (A3) and (A4). At $i=N$ and/or $j=N$, $\Phi_{i, j}$ is fixed by the following boundary conditions:

$$
\Phi_{i, N}=\Phi_{N, i}=-\frac{\tilde{M}}{4 \pi r_{i, N}}
$$

Here, $r_{i, N}=\sqrt{1+(i-1)^{2} /(N-1)^{2}} L$, and the dimensionless $^{12} \tilde{M}$ is calculated as

$$
\begin{aligned}
\tilde{M}= & \frac{\pi \delta^{3}}{4} \chi_{1,1}^{2}+\left(\sum_{i=2}^{N} 2 \pi R_{i} \delta^{2} \chi_{i, 1}^{2}\right)+\left(\sum_{j=2}^{N} \frac{\pi \delta^{3}}{2} \chi_{1, j}^{2}\right) \\
& +\sum_{i, j=2}^{N} 4 \pi R_{i} \delta^{2} \chi_{i, j}^{2},
\end{aligned}
$$

consistent with Gauss's law (see Appendix A 1 below).

Next, once $\Phi$ is fixed, the ground-state solution of Eq. (6), $\chi_{0}$, can be found by considering the following imaginary time evolution (see also Appendix B):

$$
\frac{\partial}{\partial \tau} \chi(\tau)=\nabla^{2} \chi-2\left(\Phi+\Phi_{b}\right) \chi(\tau)
$$

In the large $\tau$ limit, the asymptotic behavior of $\chi$ is

$$
\lim _{\tau \rightarrow \infty} \chi(\tau) \propto e^{-2 \gamma \tau} \chi_{0}
$$

Thus, in each iteration, $\chi_{i, j}(i, j \neq 1, N)$ is improved as

$$
\begin{aligned}
\tilde{\chi}_{i, j}= & \chi_{i, j}^{\text {old }}+\frac{\omega_{\chi} \delta^{2}}{4}\left(\frac{\chi_{i+1, j}^{\text {old }}-2 \chi_{i, j}^{\text {old }}+\chi_{i, j-1}^{\text {old }}}{\delta^{2}}+\frac{1}{R_{i}} \frac{\chi_{i+1, j}^{\text {old }}-\chi_{i-1, j}^{\text {old }}}{2 \delta}\right. \\
& \left.+\frac{\chi_{i, j+1}^{\text {old }}-2 \chi_{i, j}^{\text {old }}+\chi_{i, j-1}^{\text {old }}}{\delta^{2}}-2\left(\Phi_{i, j}+\Phi_{b, i, j}\right) \chi_{i, j}^{\text {old }}\right),
\end{aligned}
$$

\footnotetext{
${ }^{11}$ To obtain our results in this paper, we have used $\omega_{\Phi}=1.6$ in all computations. This value was chosen somewhat arbitrarily based on tests of the rate of convergence.

${ }^{12} \tilde{M}$ is related to $M$, the physical mass of the soliton, via $M=\left(\frac{M_{p l}^{2}}{4 \pi m}\right) \tilde{M}$.
} 


$$
\chi_{i, j}^{\text {new }}=\frac{\chi_{1,1}^{\text {old }}}{\tilde{\chi}_{1,1}} \tilde{\chi}_{i, j}
$$

where $\omega_{\chi}$ is an auxiliary parameter ${ }^{13}$ that we set $\mathcal{O}(1)$. For $i=1$ and/or $j=1$, the rhs of Eq. (A11) should be modified according to the prescription in Eqs. (A3) and (A4). At $i=N$ and/or $j=N, \chi_{i, j}$ is fixed by Eq. (A5).

We repeatedly update $\Phi$ and $\chi$, using Eqs. (A6) and (A11), until convergence is attained. The eigenvalue $\gamma$ is calculated as

$$
\begin{aligned}
\gamma= & \frac{-1}{2 \chi_{i, j}}\left(\frac{\chi_{i+1, j}-2 \chi_{i, j}+\chi_{i, j-1}}{\delta^{2}}+\frac{1}{R_{i}} \frac{\chi_{i+1, j}-\chi_{i-1, j}}{2 \delta}\right. \\
& \left.+\frac{\chi_{i, j+1}-2 \chi_{i, j}+\chi_{i, j-1}}{\delta^{2}}-2\left(\Phi_{i, j}+\Phi_{b, i, j}\right) \chi_{i, j}\right) .
\end{aligned}
$$

To calculate the total soliton energy, we use Eq. (10) (averaging over adjacent grid sites can be useful in order to reduce numerical error),

$$
\begin{aligned}
\tilde{E}= & \int d^{3} x\left(\frac{1}{2}(\nabla \chi)^{2}+\left(\frac{\Phi}{2}+\Phi_{b}\right) \chi^{2}\right) \\
= & \sum_{i, j=1}^{N-1} 2 \pi \delta\left(R_{i+1}^{2}-R_{i}^{2}\right) \\
& \times \frac{\left[e_{i, j}+e_{i, j+1}+e_{i+1, j}+e_{i+1, j+1}\right]}{4}
\end{aligned}
$$

with the integrand

$$
\begin{aligned}
e_{i, j}= & \frac{-\chi_{i, j}}{2}\left(\frac{\chi_{i+1, j}-2 \chi_{i, j}+\chi_{i, j-1}}{\delta^{2}}+\frac{1}{R_{i}} \frac{\chi_{i+1, j}-\chi_{i-1, j}}{2 \delta}\right. \\
& \left.+\frac{\chi_{i, j+1}-2 \chi_{i, j}+\chi_{i, j-1}}{\delta^{2}}-\left(\Phi_{i, j}+2 \Phi_{b, i, j}\right) \chi_{i, j}\right) .
\end{aligned}
$$

For $i=1$ and/or $j=1$, the rhs of Eq. (A15) should be modified according to the prescription in Eqs. (A3) and (A4). We do not need to include $i=N$ nor $j=N$ because there the integrand vanishes due to the boundary condition in Eq. (A5).

This concludes the description of the numerical scheme.

\section{Adding a black hole}

Here, we explain how a central black hole $(\mathrm{BH})$ can be added to the discretized grid calculation of Appendix A. To this end, we derive a discretized version of Gauss's law.

\footnotetext{
${ }^{13}$ To obtain our results in this paper, we have used $\omega_{\chi}=0.8$ in all computations. This value was chosen somewhat arbitrarily based on tests of the rate of convergence. We note that setting $\omega_{\chi}<\omega_{\Phi}$ appears to be useful (see footnote 11).
}

Using Eqs. (A3) and (A4), for $n<N$, we obtain

$$
\sum_{i=1}^{n} \kappa_{i} \delta\left[\left(\frac{\partial^{2}}{\partial R^{2}}+\frac{1}{R} \frac{\partial}{\partial R}\right) \Phi\right]_{i, j}=\pi(2 n-1) \frac{\Phi_{n+1, j}-\Phi_{n, j}}{\delta},
$$

$$
\sum_{j=1}^{n} \lambda_{j} \delta\left(\frac{\partial^{2} \Phi}{\partial z^{2}}\right)_{i, j}=2 \frac{\Phi_{i, n+1}-\Phi_{i, n}}{\delta}
$$

where $\kappa_{i}$ and $\lambda_{j}$ are defined as

$$
\kappa_{i}=\left\{\begin{array}{ll}
\pi / 4 & (i=1) \\
2(i-1) \pi & (i \neq 1)
\end{array}, \quad \lambda_{j}=\left\{\begin{array}{ll}
1 & (j=1) \\
2 & (j \neq 1)
\end{array} .\right.\right.
$$

From these equations, we obtain

$$
\begin{aligned}
\sum_{i=1}^{n_{i}} & \sum_{j=1}^{n_{j}} \kappa_{i} \lambda_{j} \delta^{3}\left(\nabla^{2} \Phi\right)_{i, j} \\
= & 2 \sum_{i=1}^{n_{i}} \kappa_{i} \delta^{2} \times \frac{\Phi_{i, n_{j}+1}-\Phi_{i, n_{j}}}{\delta} \\
& +2 \pi \sum_{j=1}^{n_{j}} \lambda_{j} \frac{2 n_{i}-1}{2} \delta^{2} \times \frac{\Phi_{n_{i}+1, j}-\Phi_{n_{i}, j}}{\delta}
\end{aligned}
$$

where $n_{i}, n_{j}<N$. This becomes the usual Gauss law $\int_{V} \nabla^{2} \Phi=\int_{\partial V} d S \cdot \nabla \Phi$ in the limit $\delta \rightarrow 0$.

Consider a black hole with physical mass $M_{\mathrm{BH}}$, translated in our conventions to $M_{\mathrm{BH}}=\left(M_{p l}^{2} / 4 \pi m\right) \tilde{M}_{\mathrm{BH}}$. It gives the potential $\Phi_{\mathrm{BH}}=-\tilde{M}_{\mathrm{BH}} /(4 \pi x)$. The Poisson equation is $\nabla^{2} \Phi=\rho$; thus, the discretized $\rho$ configuration that leads to $\Phi_{\mathrm{BH}}$ and that is consistent with Gauss's law, Eq. (A19), is

$$
\rho_{i, j}=\left\{\begin{array}{ll}
\frac{4 \tilde{M}_{\mathrm{BH}}}{\pi \delta^{3}} & (i=j=1) \\
0 & (i \neq 1 \quad \text { or } \quad j \neq 1)
\end{array} .\right.
$$

Finally, to interface to the code described in Appendix A, it is convenient to utilize the gravitational potential induced by the $\mathrm{BH}$, which is given on the axisymmetric grid as follows:

$$
\Phi_{\mathrm{BH}, i, j}=\left\{\begin{array}{ll}
-\frac{11 \tilde{M}_{\mathrm{BH}}}{12 \pi \delta} & (i=j=1) \\
-\frac{\tilde{M}_{\mathrm{BH}}}{4 \pi r_{i, j}} & (i \neq 1 \quad \text { or } \quad j \neq 1)
\end{array} .\right.
$$

The tricky point here is $\Phi_{\mathrm{BH}, 1,1}$; this is determined by solving the discretized Poisson equation at the origin. 


\section{APPENDIX B: IS IT THE GROUND STATE?}

In this Appendix, we present evidence that these solutions obtained by our algorithm are indeed ground-state solutions. At the same time, we also highlight the difficulty in obtaining a rigorous proof. Finally, we comment about stability to perturbations.

Let us recapitulate a few details of the imaginary time evolution of the Schrödinger equation [63-65]. The equation reads

$$
i \partial_{t} \Psi(\mathbf{r}, t)=H \Psi(\mathbf{r}, t) .
$$

The time-independent Hamiltonian $H$ is hypothesized to have eigenfunctions $\phi_{n}(\mathbf{r})$ with eigenvalues $\epsilon_{n}$, including a ground state with finite $\epsilon_{0}<0$.

Consider an initial condition

$$
\Psi(\mathbf{r}, 0)=\sum_{n} a_{n} \psi_{n}(\mathbf{r}),
$$

which can be propagated in time as

$$
\Psi(\mathbf{r}, t)=\sum_{n} e^{-i \epsilon_{n} t} a_{n} \psi_{n}(\mathbf{r}) .
$$

We can define $\tau=i t$ and rewrite the Schrödinger equation as

$$
\partial_{\tau} \tilde{\Psi}(\mathbf{r}, \tau)=-H \tilde{\Psi}(\mathbf{r}, \tau),
$$

with the initial condition

$$
\tilde{\Psi}(\mathbf{r}, 0)=\sum_{n} a_{n} \psi_{n}(\mathbf{r})
$$

and a general solution

$$
\tilde{\Psi}(\mathbf{r}, \tau)=\sum_{n} e^{-\epsilon_{n} \tau} a_{n} \psi_{n}(\mathbf{r}) .
$$

In the limit $\tau \rightarrow \infty$, we have

$$
\lim _{\tau \rightarrow \infty} \tilde{\Psi}(\mathbf{r}, \tau)=e^{-\epsilon_{0} \tau} a_{0} \psi_{0}(\mathbf{r}),
$$

thus providing the sought-after ground state, $\psi_{0}$.

The difficulty in this formalism, which becomes apparent in the regime where the self-gravitation is dynamically relevant, is that the Hamiltonian is not constant between iterations but rather changes as we iterate on the wave function and the Newtonian potential induced by it. Thus, while the solutions found by our solver are (within the numerical accuracy) indeed solutions of the EOM, we have no rigorous proof that these are in fact the ground-state solutions. Having made this cautionary remark, we now present some evidence that our solution is indeed the ground state, at least when it comes to ULDM in the background baryonic potential of realistic galaxies.

The first thing to note is that in the limit at which selfgravity is negligible compared to the external potential the problem becomes linear, the Hamiltonian is constant, and the derivation leading to Eq. (B7) is applicable without particular complications. Then, the formalism leading to
Eq. (B7) suggests that our solution does indeed isolate the true ground state, as long as the initial test function has some nonvanishing overlap with this ground state. In specific examples, we can compare the numerical results to analytic solutions. The case of a strong baryonic potential concentrated near the origin is a good example; in this case, the exact solution converges to the Coulomb wave function $\chi(r) \propto e^{-A r}$.

On the other hand, in the opposite limit where the external potential is negligible and self-gravity dominates, we find that our algorithm converges to the known selfgravitating ground-state solution.

Many examples in the paper (e.g., Figs. 1, 5-9) explicitly examine the behavior of the solution while going smoothly between the two limits of negligible external potential and all the way to where the external potential dominates the solution. The two limits are smoothly connected by a continuous deformation. This lends support to the notion that also in the intermediate regime our solver is finding the true ground-state solution.

We have also made sure that the solutions are not sensitive to the details of the test function used as the initial condition. For a given external potential $\Phi_{b}$, we checked a variety of initial conditions of the field $\chi_{i, j}$, including Gaussian forms with different slopes as well as randomized independent realizations of the field on different grid points [always keeping $\chi_{i=N, j}=\chi_{i, j=N}=0$ as prescribed in Eq. (A5)]. For some of these initial conditions, the solver converges on a solution, while for others, it does not. Importantly, whenever the solver does converge, the different initial conditions all lead to the same solution. Note that the solver sometimes does not converge when the initial conditions do not fall steeply enough as a function of distance away from the origin. In addition, convergence also shows some dependence on the function $\Phi_{b}$ used in the test. A simple choice which works well for all of the problems we experimented with was to use the spherical self-gravitating solution as the initial test function.

We now give a short comment about the linear stability of our solutions. It is useful to first recall the stability argument for the self-gravitating soliton: in the Newtonian limit, the mass and the energy of the field are conserved separately; since the soliton is the field configuration that minimizes the energy at a fixed value of the mass, it is guaranteed on general grounds to be dynamically stable [7]. ${ }^{14}$ Once we "turn on" an external potential (spherically symmetric or not), however, linear stability could become a concern.

Our solver finds solutions of the EOM while holding the value of the field fixed at the origin [via the $\chi(0)=\lambda^{2}$ prescription]. Let us assume, based on the arguments given in the previous paragraphs, that the solution we find is indeed the lowest energy solution compatible with the

\footnotetext{
${ }^{14}$ Relativistic corrections do cause soliton decay [21,24], but the decay time is long and of no phenomenological relevance in the range of ULDM and soliton masses considered in this work.
} 
boundary conditions. One can show that in the limit where the external potential dominates the solution, the boundary condition $\chi(0)$ remains in a monotonous one-to-one correspondence to the mass of the solution as long as the external potential decays away from the origin. Therefore, we expect that our solutions remain minimizers of the ULDM energy at fixed mass, as long as the external potential does not exhibit strong departure from monotonous decay (this assumption is satisfied for the LSB and MW examples that we analysed in the main text). This settles the stability question for the limit where the external potential strongly dominates; as long as the external potential is static and does not rearrange itself dynamically following a change in the ULDM system, there is no energy exchange between the ULDM and the external system, and the soliton stability is guaranteed.

The intermediate limit, where the external potential is comparable to that coming from the ULDM, is much more complicated. Addressing the question of stability in this case requires a joint analysis of the baryonic system and the ULDM. This analysis is beyond the scope for the current paper. Indeed, our goal in this work is not to solve the (in general, very difficult) dynamical problem of finding stable gravitating solutions of the joint baryonic and ULDM system. Instead, our starting point is to assume that the baryonic part of the system is already known via observational constraints like stellar luminosity and gas line emission (as was the case for the LSB galaxies we analyzed) and then derive the minimum energy soliton consistent with this known external background.

\section{APPENDIX C: GRAVITATIONAL POTENTIAL OF AN AXISYMMETRIC MASS DISTRIBUTION}

The solution of the Poisson equation in axisymmetry can be found directly using the method of the Fourier-Bessel transform. Following Ref. [66], the gravitational potential is given by

$\phi(R, z)=-2 \pi G \int_{-\infty}^{\infty} d \zeta \int_{0}^{\infty} d u \rho(u, \zeta) K(R, u,|z-\zeta|)$,

where the kernel $K$ is given by

$$
\begin{aligned}
K(R, u, z) & =u \int_{0}^{\infty} d k J_{0}(k R) J_{0}(k u) e^{-k z} \\
& =\frac{\sqrt{u}}{\pi \sqrt{R}} \operatorname{Re}\left[Q_{-\frac{1}{2}}\left(\frac{R^{2}+u^{2}+z^{2}}{2 R u}\right)\right]
\end{aligned}
$$

with $Q_{-\frac{1}{2}}$ the Legendre function of the second kind of order $-\frac{1}{2}$. See also 6.612 (3) and 8.834 (1) in Ref. [67].

\section{APPENDIX D: MODELING STELLAR DISKS}

In order to simplify the analysis, we take advantage of the Miyamoto-Nagai (MN) [68] disk parametrization, described by the density profile

$$
\begin{aligned}
\rho_{\mathrm{MN}}(R, z)= & \frac{M_{\mathrm{MN}} b^{2}}{4 \pi} \\
& \times \frac{a R^{2}+\left(a+3 \sqrt{z^{2}+b^{2}}\right)\left(a+\sqrt{z^{2}+b^{2}}\right)^{2}}{\left(R^{2}+\left(a+\sqrt{z^{2}+b^{2}}\right)^{2}\right)^{5 / 2}\left(z^{2}+b^{2}\right)^{3 / 2}}
\end{aligned}
$$

for which the gravitational potential is known analytically:

$$
\phi_{\mathrm{MN}}(R, z)=-\frac{G M_{\mathrm{MN}}}{\sqrt{R^{2}+\left(a+\sqrt{z^{2}+b^{2}}\right)^{2}}} .
$$

The parameters $a, b$, and $M_{\mathrm{MN}}$ define the disk scale radius, thickness, and mass. A sum of three MN profiles provides a reasonable approximation to the exponential disks of typical galaxies [69].

In the SPARC database, the surface brightness $\Sigma_{L}(R)$ of the disk component is reported. Assuming an exponential approximation for the vertical direction, the stellar mass density is given by

$$
\rho(R, z)=\Upsilon_{d} \Sigma_{L}(R) \frac{e^{-\frac{|z|}{z_{d}}}}{2 z_{d}}
$$

where $z_{d}$ is specified in the database for each galaxy. One can then fit the MN density on the disk plane,

$$
\rho_{\mathrm{MN}}(R, 0)=\frac{M_{\mathrm{MN}}\left[a R^{2}+(a+3 b)(a+b)^{2}\right]}{4 \pi b\left[R^{2}+(a+b)^{2}\right]^{5 / 2}}
$$

(or a sum of such functions), to

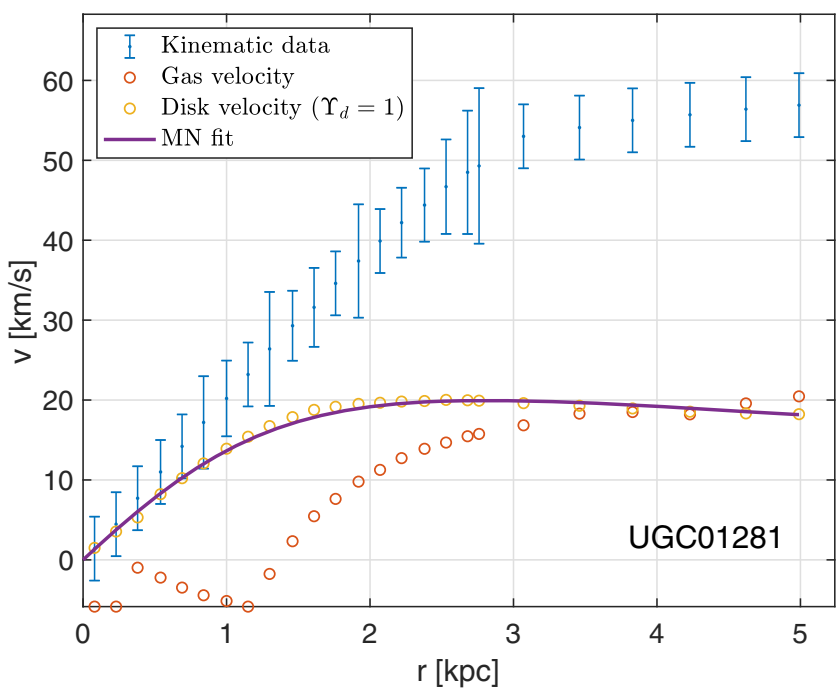

FIG. 11. MN fit for the disk of UGC01281. The disk fit is shown by the solid purple line, while the orange circles show the velocity attributed to the disk in the SPARC database for $\Upsilon_{d}=1$. 


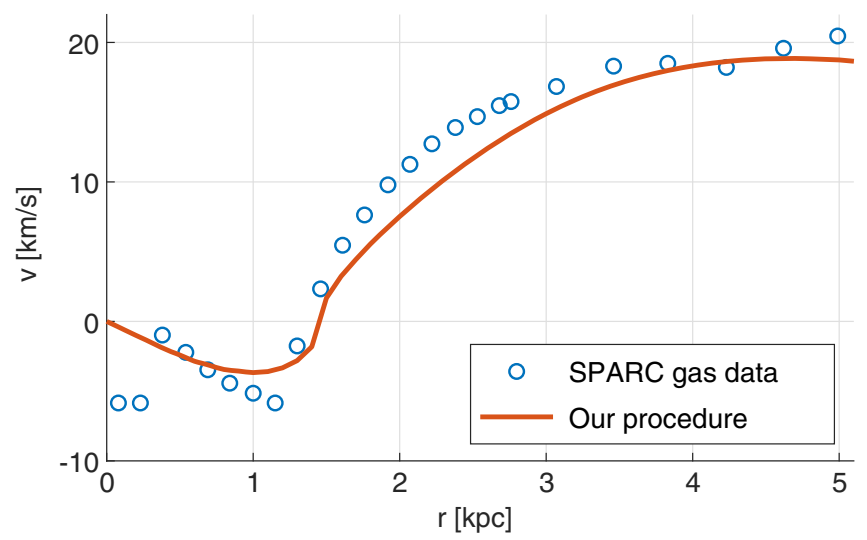

FIG. 12. Modeling the gas contribution to the rotation curve of UGC01281.

$$
\rho(R, 0)=\frac{\Upsilon_{d} \Sigma_{L}(R)}{2 z_{d}}
$$

fixing $b=z_{d}$.

In Fig. 11, we show the rotation curve decomposition for UGC01281, superimposed with a MN fit for the disk obtained with the above prescription.

\section{APPENDIX E: MODELING THE GAS DISTRIBUTION IN UGC01281}

The SPARC database [31] does not contain sufficient information to allow a direct reconstruction of the gas mass distribution. ${ }^{15}$ We have therefore done an independent analysis of the gas component for the example of UGC01281, using the HI surface brightness profiles reported in Ref. [59].

Our analysis is less sophisticated than that in Ref. [59] but captures the key features of the gas profile with sufficient accuracy. We model the gas density profile as a collection of $K$ coplanar rings, with the mass density of each ring taking to be constant on the plane $(z=0)$ and decaying vertically with a Gaussian profile,

$\rho_{\text {gas }}(R, z)=\sum_{k=1}^{K} \theta\left(R-R_{k}\right) \theta\left(R_{k}+\Delta_{k}-R\right) \rho_{k} e^{-\frac{z^{2}}{d_{z}^{2}}}$,

where $\theta(x)$ is the Heaviside function. The gravitational potential due to this mass distribution is computed by the procedure given in Appendix C.

The surface-brightness profile from this gas distribution is easily computed. Matching the model to the vertical profile reported in Ref. [59], we find a good fit for $d_{z}=0.65 \mathrm{kpc}$. Considering the radial profile and matching (approximately, by eye) to the average profile shown in Fig. 2 of Ref. [59] (which averages the HI column density over a slab in the vertical direction), we find that a model of $K=50$ rings of equal width $\Delta_{k}=0.2 \mathrm{kpc}$, located with

\footnotetext{
${ }^{15}$ We thank Stacy McGaugh for clarification on this point.
}

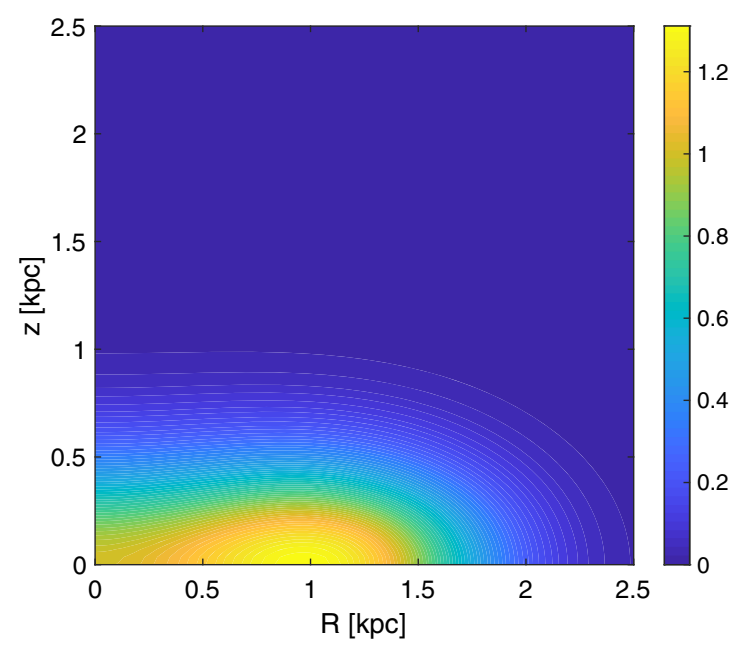

FIG. 13. Density profile of a toroidal soliton solution.
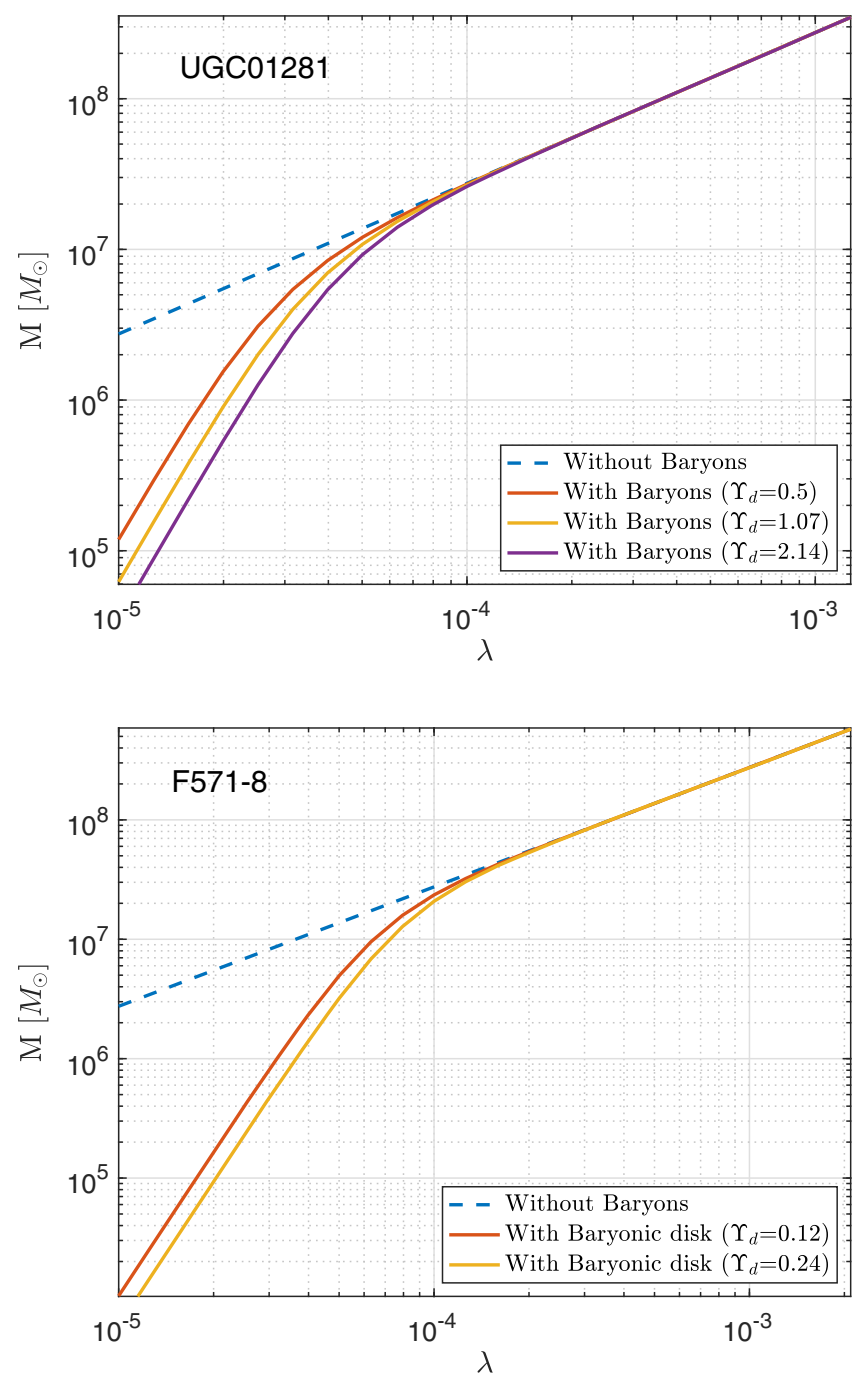

FIG. 14. Soliton $\mathrm{M}-\lambda$ relation in baryonic-induced background gravitational potential of SPARC galaxies. Top panel: UGC01281. Bottom panel: 571-8. The ULDM particle mass is $m=10^{-21} \mathrm{eV}$. 

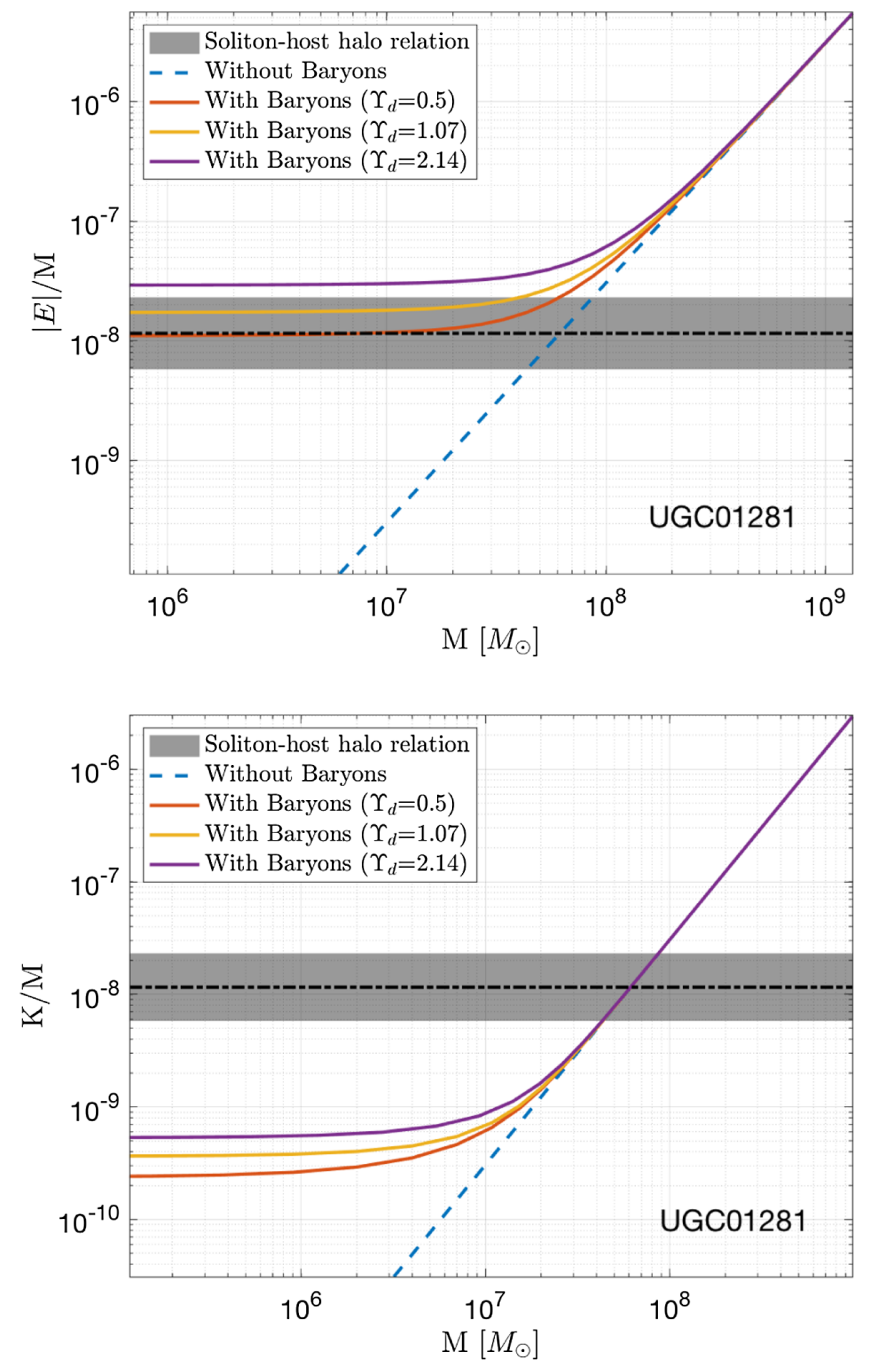

FIG. 15. Specific energy $|E| / M$ (top) and specific kinetic energy $K / M$ (bottom) for a soliton in UGC01281. The Soliton-Host Halo Relation found in DM-only numerical simulations is shown by the black dashed line with a shaded band denoting a factor of 2 estimated spread. The ULDM particle mass is $m=10^{-21} \mathrm{eV}$.

inner radii starting at $R_{1}=0 \mathrm{kpc}$ up to $R_{50}=10 \mathrm{kpc}$, reproduces the brightness profile radial shape for the density assignment $\rho_{k}=\tilde{\rho}\left(0.5+R_{k}\right)^{1.2} \exp \left(-\left(\frac{R_{k}}{1.5}\right)^{1.4}\right)$, where $R_{k}$ are noted in kiloparsecs and $\tilde{\rho}$ is an overall normalization factor. We set $\tilde{\rho}=3.9 \times 10^{6} M_{\odot} / \mathrm{kpc}^{3}$, so that the total gas mass (including a factor of 1.3 to account for $\mathrm{He}$ ) is fixed to $M_{\mathrm{gas}}=3.2 \times 10^{8} M_{\odot}$, inferred in Ref. [60] from the total HI luminosity.

The gas-induced rotation curve we find with this procedure is shown by the line in Fig. 12, compared to the velocity contribution attributed to the gas in the SPARC database (circles). The comparison is good enough for our purpose in the current work; as we show in the body of the work, the total baryonic effect (stars and gas combined) on the predicted soliton and on the large-scale halo of UGC01281 is small.
We conclude this technical discussion with an amusing comment. The toroidal gas profile of UGC01281 prompted us to look for toroidal soliton solutions that could coexist in the background potential of such a baryonic mass distribution. Indeed, varying the gas mass and the soliton mass, we can find toroidal solitons; we show an example in Fig. 13. The parameters chosen to achieve this toroidal solution were $m=10^{-22} \mathrm{eV}$, with $\lambda=10^{-5}$ and a gas mass 50 times larger than the observed one in UGC01281. These parameters do not represent an actual galaxy from SPARC; we merely bring it as an observation about deformed solitons and as a demonstration of the versatility of the numerical code.

\section{APPENDIX F: RESULTS WITH ULDM PARTICLE MASS OF $m=10^{-21} \mathrm{eV}$}

Figs. 14-16 present a repetition of results from Sec. IV, done for ULDM particle mass $m=10^{-21} \mathrm{eV}$.
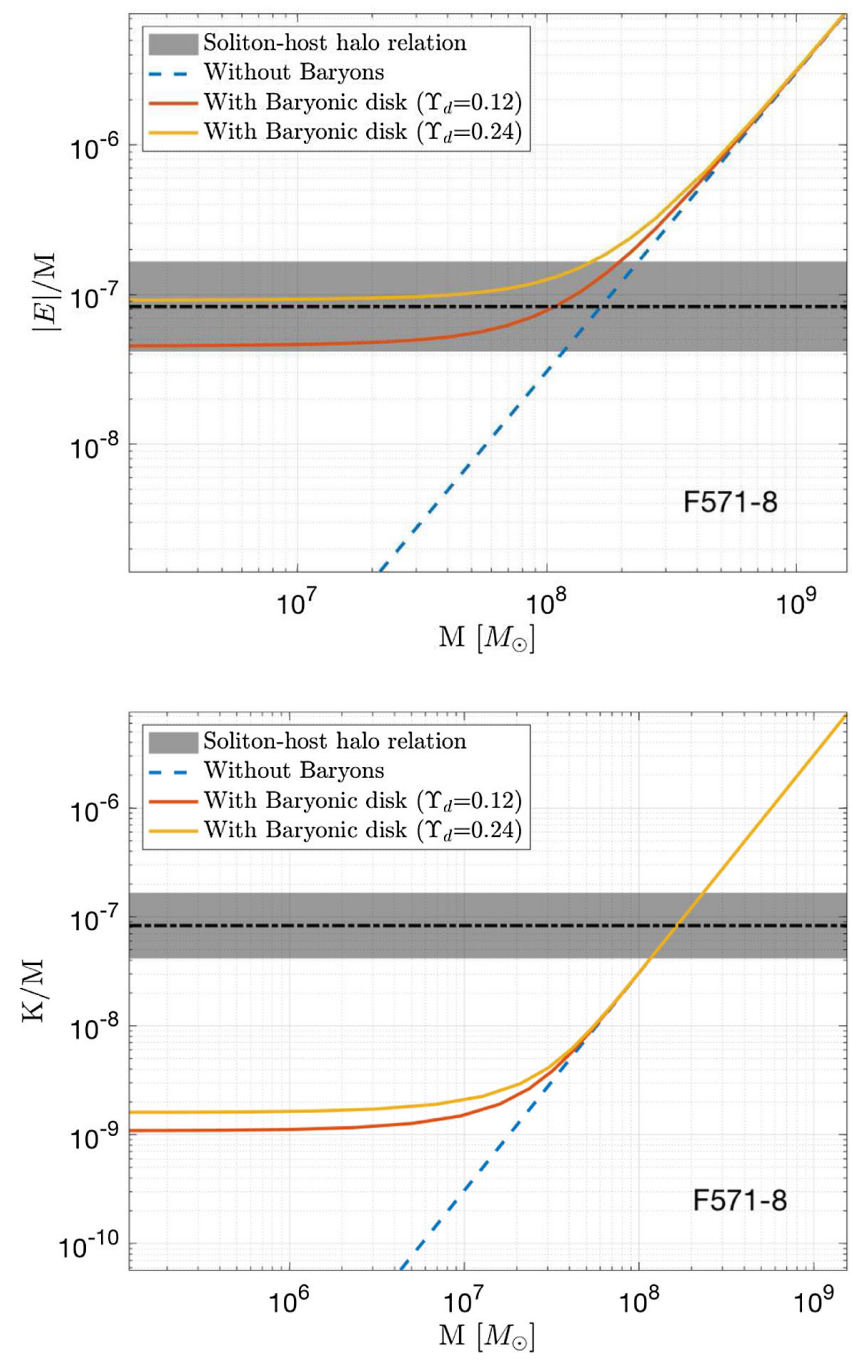

FIG. 16. Same as Fig. 15, but done for F571-8. 
[1] W. Hu, R. Barkana, and A. Gruzinov, Cold and Fuzzy Dark Matter, Phys. Rev. Lett. 85, 1158 (2000).

[2] P. Svrcek and E. Witten, Axions in string theory, J. High Energy Phys. 06 (2006) 051.

[3] A. Arvanitaki, S. Dimopoulos, S. Dubovsky, N. Kaloper, and J. March-Russell, String Axiverse, Phys. Rev. D 81, 123530 (2010).

[4] D. J. E. Marsh, Axion Cosmology, Phys. Rep. 643, 1 (2016).

[5] A. Arbey, J. Lesgourgues, and P. Salati, Quintessential haloes around galaxies, Phys. Rev. D 64, 123528 (2001).

[6] J. Lesgourgues, A. Arbey, and P. Salati, A light scalar field at the origin of galaxy rotation curves, New Astron. Rev. 46, 791 (2002).

[7] P.-H. Chavanis, Mass-radius relation of Newtonian selfgravitating Bose-Einstein condensates with short-range interactions: I. Analytical results, Phys. Rev. D 84, 043531 (2011).

[8] P. H. Chavanis and L. Delfini, Mass-radius relation of Newtonian self-gravitating Bose-Einstein condensates with short-range interactions: II. Numerical results, Phys. Rev. D 84, 043532 (2011).

[9] H.-Y. Schive, T. Chiueh, and T. Broadhurst, Cosmic structure as the quantum interference of a coherent dark wave, Nat. Phys. 10, 496 (2014).

[10] H.-Y. Schive, M.-H. Liao, T.-P. Woo, S.-K. Wong, T. Chiueh, T. Broadhurst, and W. Y. P. Hwang, Understanding the Core-Halo Relation of Quantum Wave Dark Matter from 3D Simulations, Phys. Rev. Lett. 113, 261302 (2014).

[11] D. J. E. Marsh and A.-R. Pop, Axion dark matter, solitons and the cusp-core problem, Mon. Not. R. Astron. Soc. 451, 2479 (2015).

[12] E. Calabrese and D. N. Spergel, Ultra-light dark matter in ultra-faint dwarf galaxies, Mon. Not. R. Astron. Soc. 460, 4397 (2016).

[13] S.-R. Chen, H.-Y. Schive, and T. Chiueh, Jeans analysis for dwarf spheroidal galaxies in wave dark matter, Mon. Not. R. Astron. Soc. 468, 1338 (2017).

[14] B. Schwabe, J. C. Niemeyer, and J. F. Engels, Simulations of solitonic core mergers in ultralight axion dark matter cosmologies, Phys. Rev. D 94, 043513 (2016).

[15] J. Veltmaat and J. C. Niemeyer, Cosmological particle-incell simulations with ultralight axion dark matter, Phys. Rev. D 94, 123523 (2016).

[16] L. Hui, J. P. Ostriker, S. Tremaine, and E. Witten, Ultralight scalars as cosmological dark matter, Phys. Rev. D 95, 043541 (2017).

[17] A. X. Gonzáles-Morales, D. J. E. Marsh, J. Peñarrubia, and L. U. López, Unbiased constraints on ultralight axion mass from dwarf spheroidal galaxies, Mon. Not. R. Astron. Soc. 472, 1346 (2017).

[18] V. H. Robles and T. Matos, Flat central density profile and constant DM surface density in galaxies from scalar field dark matter, Mon. Not. R. Astron. Soc. 422, 282 (2012).

[19] T. Bernal, L. M. Fernández-Hernández, T. Matos, and M. A. Rodrguez-Meza, Rotation curves of high-resolution LSB and SPARC galaxies in wave (fuzzy) and multistate (ultralight boson) scalar field dark matter, Mon. Not. R. Astron. Soc. 475, 1447 (2018).
[20] P. Mocz, M. Vogelsberger, V. H. Robles, J. Zavala, M. Boylan-Kolchin, A. Fialkov, and L. Hernquist, Galaxy formation with BECDM: I. Turbulence and relaxation of idealized haloes, Mon. Not. R. Astron. Soc. 471, 4559 (2017).

[21] K. Mukaida, M. Takimoto, and M. Yamada, On longevity of I-ball/oscillon, J. High Energy Phys. 03 (2017) 122.

[22] J. Vicens, J. Salvado, and J. Miralda-Escudé, Bosonic dark matter halos: Excited states and relaxation in the potential of the ground state, arXiv:1802.10513.

[23] N. Bar, D. Blas, K. Blum, and S. Sibiryakov, Galactic rotation curves versus ultralight dark matter: Implications of the soliton-host halo relation, Phys. Rev. D 98, 083027 (2018).

[24] J. Eby, K. Mukaida, M. Takimoto, L. C. R. Wijewardhana, and M. Yamada, Classical nonrelativistic effective field theory and the role of gravitational interactions, arXiv: 1807.09795.

[25] B. Bar-Or, J.-B. Fouvry, and S. Tremaine, Relaxation in a Fuzzy Dark Matter Halo, Astrophys. J. 871, 28 (2019).

[26] D. J. E. Marsh and J. C. Niemeyer, Strong constraints on fuzzy dark matter from ultrafaint dwarf galaxy eridanus II, arXiv: 1810.08543.

[27] P.-H. Chavanis, A predictive model of BEC dark matter halos with a solitonic core and an isothermal atmosphere, arXiv: 1810.08948 .

[28] R. Emami, T. Broadhurst, G. Smoot, T. Chiueh, and L. H. Nhan, A soliton solution for the central dark masses in globular clusters and implications for the axiverse, arXiv:1806.04518.

[29] D. G. Levkov, A. G. Panin, and I. I. Tkachev, Gravitational Bose-Einstein Condensation in the Kinetic Regime, Phys. Rev. Lett. 121, 151301 (2018).

[30] T. Broadhurst, I. de Martino, H. N. Luu, G. F. Smoot, and S. H. H. Tye, Ghostly galaxies as solitons of Bose-Einstein dark matter, arXiv:1902.10488.

[31] F. Lelli, S. S. McGaugh, and J. M. Schombert, SPARC: Mass models for 175 disk galaxies with Spitzer photometry and accurate rotation curves, Astron. J. 152, 157 (2016).

[32] J. Veltmaat, J. C. Niemeyer, and B. Schwabe, Formation and structure of ultralight bosonic dark matter halos, Phys. Rev. D 98, 043509 (2018).

[33] H. Deng, M. P. Hertzberg, M. H. Namjoo, and A. Masoumi, Can light dark matter solve the core-cusp problem, Phys. Rev. D 98, 023513 (2018).

[34] E. Armengaud, N. Palanque-Delabrouille, D. J. E. Marsh, J. Baur, and C. Yche, Constraining the mass of light bosonic dark matter using SDSS Lyman- $\alpha$ forest, Mon. Not. R. Astron. Soc. 471, 4606 (2017).

[35] V. Iršič, M. Viel, M. G. Haehnelt, J. S. Bolton, and G. D. Becker, First Constraints on Fuzzy Dark Matter from Lyman- $\alpha$ Forest Data and Hydrodynamical Simulations, Phys. Rev. Lett. 119, 031302 (2017).

[36] J. Zhang, J.-L. Kuo, H. Liu, Y.-L. S. Tsai, K. Cheung, and M.-C. Chu, Is fuzzy dark matter in tension with lyman-alpha forest, Astrophys. J. 863, 73 (2018).

[37] T. Kobayashi, R. Murgia, A. De Simone, V. Iršič, and M. Viel, Lyman-alpha constraints on ultralight scalar dark matter: Implications for the early and late universe, Phys. Rev. D 96, 123514 (2017). 
[38] K.-H. Leong, H.-Y. Schive, U.-H. Zhang, and T. Chiueh, Testing extreme-axion wave dark matter using the BOSS Lyman-Alpha forest data, Mon. Not. R. Astron. Soc. 484, 4273 (2019).

[39] B. Bozek, D. J. E. Marsh, J. Silk, and R. F. G. Wyse, Galaxy UV-luminosity function and reionization constraints on axion dark matter, Mon. Not. R. Astron. Soc. 450, 209 (2015).

[40] R. Hlozek, D. J. E. Marsh, and D. Grin, Using the full power of the cosmic microwave background to probe axion dark matter, Mon. Not. R. Astron. Soc. 476, 3063 (2018).

[41] A. Khmelnitsky and V. Rubakov, Pulsar timing signal from ultralight scalar dark matter, J. Cosmol. Astropart. Phys. 02 (2014) 019.

[42] N. K. Porayko et al., Parkes pulsar timing array constraints on ultralight scalar-field dark matter, Phys. Rev. D 98, 102002 (2018).

[43] B. V. Church, J. P. Ostriker, and P. Mocz, Heating of milky way disc stars by dark matter fluctuations in cold dark matter and fuzzy dark matter Paradigms, Mon. Not. R. Astron. Soc. 485, 2861 (2019).

[44] N. C. Amorisco and A. Loeb, First constraints on fuzzy dark matter from the dynamics of stellar streams in the milky way, arXiv:1808.00464.

[45] A. Schneider, Constraining non-cold dark matter models with the global 21-cm signal, Phys. Rev. D 98, 063021 (2018).

[46] J. D. Bowman, A. E. E. Rogers, R. A. Monsalve, T. J. Mozdzen, and N. Mahesh, An absorption profile centred at 78 megahertz in the sky-averaged spectrum, Nature (London) 555, 67 (2018).

[47] A. Del Popolo and M. Le Delliou, Small scale problems of the $\Lambda$ CDM model: A short review, Galaxies 5, 17 (2017).

[48] J. H. H. Chan, H.-Y. Schive, T.-P. Woo, and T. Chiueh, How do stars affect $\psi$ DM halos, arXiv:1712.01947.

[49] K. Hayashi and I. Obata, Non-sphericity of ultra-light axion dark matter halos in the Galactic dwarf spheroidal galaxies, arXiv:1902.03054.

[50] S. Alexander, J. J. Bramburger, and E. McDonough, Novel substructure and superfluid dark matter, arXiv:1901.03694.

[51] T. Rindler-Daller and P. R. Shapiro, Finding new signature effects on galactic dynamics to constrain Bose-EinsteinCondensed cold dark matter, in Accelerated Cosmic Expansion. Astrophysics and Space Science Proceedings, edited by C. Moreno González, J. Madriz Aguilar, and L. Reyes Barrera (Springer, Cham, 2014), Vol. 38.

[52] V. Desjacques, A. Kehagias, and A. Riotto, Impact of ultralight axion self-interactions on the large scale structure of the Universe, Phys. Rev. D 97, 023529 (2018).

[53] R. Ruffini and S. Bonazzola, Systems of selfgravitating particles in general relativity and the concept of an equation of state, Phys. Rev. 187, 1767 (1969).

[54] R. Launhardt, R. Zylka, and P. G. Mezger, The nuclear bulge of the galaxy. 3. Large scale physical characteristics of stars and interstellar matter, Astron. Astrophys. 384, 112 (2002).

[55] R. Schönrich, M. Aumer, and S. E. Sale, Kinematic detection of the galactic nuclear disk, Astrophys. J. Lett. 812, L21 (2015).

[56] M. Portail, O. Gerhard, C. Wegg, and M. Ness, Dynamical modelling of the galactic bulge and bar: The Milky Way's pattern speed, stellar and dark matter mass distribution, Mon. Not. R. Astron. Soc. 465, 1621 (2017).

[57] C. Wegg, O. Gerhard, and M. Portail, The initial mass function of the inner galaxy measured from OGLEIII microlensing timescales, Astrophys. J. Lett. 843, L5 (2017).

[58] I. De Martino, T. Broadhurst, S. H. H. Tye, T. Chiueh, and H.-Y. Schive, Dynamical evidence of a solitonic core of $10^{9} M_{\odot}$ in the Milky Way, arXiv:1807.08153.

[59] P. Kamphuis, R. F. Peletier, P. C. van der Kruit, and G. H. Heald, Warp or lag? The ionized and neutral hydrogen gas in the edge-on dwarf galaxy UGC 1281, Mon. Not. R. Astron. Soc. 414, 3444 (2011).

[60] W. J. G. de Blok and A. Bosma, High-resolution rotation curves of low surface brightness galaxies, Astron. Astrophys. 385, 816 (2002).

[61] N. Starkman, F. Lelli, S. McGaugh, and J. Schombert, A new algorithm to quantify maximum discs in galaxies, Mon. Not. R. Astron. Soc. 480 (2018).

[62] W. H. Press, S. A. Teukolsky, W. T. Vetterling, and B. P. Flannery, Numerical Recipes in C: The Art of Scientific Computing 2nd ed. (Cambridge University Press, Cambridge, England, 1992).

[63] R. Grimm and R. G. Storer, A new method for the numerical solution of the schrödinger equation, J. Comput. Phys. 4, 230 (1969).

[64] A. Goldberg and J.L. Schwartz, Integration of the schrödinger equation in imaginary time. i., J. Comput. Phys. 1, 433 (1967).

[65] A. Goldberg and J.L. Schwartz, Integration of the schrödinger equation in imaginary time. ii., J. Comput. Phys. 1, 448 (1967).

[66] S. Casertano, Rotation curve and mass model for the edge-on galaxy ngc 5907, Internal Kinematics and Dynamics of Galaxies (Springer, Dordrecht, Netherlands, 1983).

[67] I. S. Gradshteyn and I. M. Ryzhik, Table of Integrals, Series, and Products (Academic, New York, 1980).

[68] M. Miyamoto and R. Nagai, Three-dimensional models for the distribution of mass in galaxies, Publ. Astron. Soc. Jpn. 27, 533 (1975).

[69] R. Smith, C. Flynn, G. N. Candlish, M. Fellhauer, and B. K. Gibson, Simple and accurate modelling of the gravitational potential produced by thick and thin exponential discs, Mon. Not. R. Astron. Soc. 448, 2934 (2015). 